\title{
Gravitational waves and electroweak baryogenesis in a global study of the extended scalar singlet model
}

\author{
Ankit Beniwal, ${ }^{a, b, 1}$ Marek Lewicki, ${ }^{b, c, d, 2}$ Martin White $^{b}$ and Anthony G. Williams ${ }^{b, 3}$ \\ ${ }^{a}$ The Oskar Klein Centre for Cosmoparticle Physics, Department of Physics, Stockholm University, \\ AlbaNova, SE-106 91 Stockholm, Sweden \\ ${ }^{b}$ ARC Centre of Excellence for Particle Physics at the Terascale (CoEPP) and CSSM, \\ Department of Physics, University of Adelaide, \\ South Australia 5005, Adelaide, Australia \\ ${ }^{c}$ Kings College London, Strand, London, WC2R 2LS, U.K. \\ ${ }^{d}$ Faculty of Physics, University of Warsaw, \\ ul. Pasteura 5, 02-093 Warsaw, Poland \\ E-mail: ankit.beniwal@fysik.su.se, marek.lewicki@kcl.ac.uk, \\ martin.white@adelaide.edu.au, anthony.williams@adelaide.edu.au
}

ABSTRACT: We perform a global fit of the extended scalar singlet model with a fermionic dark matter (DM) candidate. Using the most up-to-date results from the Planck measured DM relic density, direct detection limits from the XENON1T (2018) experiment, electroweak precision observables and Higgs searches at colliders, we constrain the 7dimensional model parameter space. We also find regions in the model parameter space where a successful electroweak baryogenesis (EWBG) can be viable. This allows us to compute the gravitational wave (GW) signals arising from the phase transition, and discuss the potential discovery prospects of the model at current and future GW experiments. Our global fit places a strong upper and lower limit on the second scalar mass, the fermion DM mass and the scalar-fermion DM coupling. In agreement with previous studies, we find that our model can simultaneously yield a strong first-order phase transition and saturate the observed DM abundance. More importantly, the GW spectra of viable points can often be within reach of future GW experiments such as LISA, DECIGO and BBO.

Keywords: Beyond Standard Model, Higgs Physics, Spontaneous Symmetry Breaking, Thermal Field Theory

ArXiv EPrint: 1810.02380

\footnotetext{
${ }^{1}$ ORCID ID: 0000-0003-4849-0611.

${ }^{2}$ ORCID ID: 0000-0002-8378-0107.

${ }^{3}$ ORCID ID: 0000-0002-1472-1592.
} 


\section{Contents}

1 Introduction 1

2 Singlet fermion dark matter model $\quad 3$

3 Constraints and likelihoods $\quad 4$

3.1 Thermal relic density 6

3.2 Direct detection 8

$\begin{array}{llr}3.3 & \text { Electroweak baryogenesis (EWBG) } & 9\end{array}$

$\begin{array}{ll}3.4 & \text { Electroweak precision observables (EWPO) } \\ 3.5 & \text { Higgs sear }\end{array}$

$\begin{array}{ll}3.5 & \text { Higgs searches at colliders } \\ \end{array}$

4 Results $\quad 14$

$\begin{array}{lll}\text { 4.1 EWBG only } & 15\end{array}$

$\begin{array}{lll}4.2 \text { Global fit } & 17\end{array}$

$\begin{array}{lll}\text { 4.2.1 Scenario I: } f_{\text {rel }} \leq 1 & 17\end{array}$

4.2.2 Scenario II: $f_{\text {rel }}=1 \quad 21$

$\begin{array}{lll}4.3 & \text { Gravitational wave signals } & 22\end{array}$

5 Conclusions $\quad 26$

$\begin{array}{ll}\text { A Tree-level scalar potential } & 27\end{array}$

$\begin{array}{ll}\text { B Mass eigenstate basis } & 29\end{array}$

$\begin{array}{ll}\text { C Dark matter-nucleon coupling } & 31\end{array}$

D Effective potential $\quad 32$

\section{Introduction}

The discovery of the Higgs boson at the LHC [1,2] has finally completed the Standard Model (SM) of particle physics. Not only does it provide a new way to study the properties of the Higgs boson, it also offers a way to investigate the details of electroweak symmetry breaking (EWSB). Meanwhile, a more recent observation of the first gravitational wave (GW) signal [3] and subsequent discoveries [4-10] have opened up a new window to probe the early history of our universe. In particular, rather violent events such as the firstorder electroweak phase transition (EWPT) would necessarily leave GW imprints. With the current and future generations of ground/space-based GW experiments, we can hope to observe such signals [11-13]. The existence of dark matter (DM) also offers a way to 
probe the early history of our universe. With the current generation of direct DM searches, experiments are probing the DM-nucleon interaction with increasing sensitivity and placing strong limits on the allowed particle DM models.

Motivated by the above experimental probes that are constantly developing, we revisit an extended scalar singlet extension of the SM in this paper. In particular, we focus on two main features of this model. Firstly, it helps to facilitate electroweak baryogenesis (EWBG) [14-17], a mechanism that aims to explain the observed matter-antimatter asymmetry via a strong first-order EWPT. In the SM, this phase transition is not firstorder $[18,19]$ and thus requires a modification. With an extra scalar, a potential barrier can be generated between the symmetric high-temperature minimum and the EWSB one as the universe cools down $[20,21]$. This leads to a strong first-order EWPT which can be probed using GWs and standard collider searches [22-42]. Secondly, the new scalar mixes with the SM Higgs boson and provides a portal for a fermion DM to saturate the observed DM abundance [43-45].

Simple DM models with a Higgs portal type interaction are still viable and enjoy a rich interest in the particle physics community [43-60]. In our study, we focus on a singlet fermion DM model which was first introduced in ref. [61] and subsequently improved in ref. [62]. After the discovery of a SM-like Higgs boson at the LHC, the model was revisited in ref. [63] in the context of vacuum stability (see also ref. [64]). Here it was pointed out that the model is stable and perturbative up to the Planck scale for a $125 \mathrm{GeV}$ Higgs boson. In light of EWBG, the model was first studied in ref. [65] and more recently in ref. [66]. Using a Monte Carlo scan of the model parameter space, the model was shown to realise a strong first-order phase transition without conflicting with any bounds from direct DM searches, electroweak precision observables (EWPO) and latest Higgs data from the LHC.

We aim to perform the most comprehensive and up-to-date study of the extended scalar singlet model with a fermionic DM candidate. In our global fit, we include the latest results from the Planck measured DM relic density [67], direct detection limits from the XENON1T (2018) experiment [68], EWPO [69] and Higgs searches at colliders [70, 71]. We also find regions in the model parameter space where a successful EWBG can be viable, compute the resulting GW spectra, and check the discovery prospects of the model at current and future GW experiments. In agreement with previous studies, we confirm that our model with additional couplings to the SM Higgs boson can simultaneously explain the observed DM abundance and matter-antimatter asymmetry; this was not possible in the $\mathbb{Z}_{2}$ symmetric case studied in our previous work [36]. We also find that our global fit places a strong upper and lower limit on the second scalar mass $m_{H}$, fermion DM mass $m_{\psi}$ and the scalar-fermion DM coupling $g_{S}$. In addition, the GW spectra of viable points can often be within reach of future GW experiments such LISA, DECIGO and BBO.

The rest of the paper is organised as follows. In section 2, we introduce the extended scalar singlet model with a fermionic DM candidate. After taking note of the free parameters of our model, we describe a set of constraints and likelihoods used in our global fit in section 3. Our model results and conclusions are presented in sections 4 and 5 respectively. Appendices A, B, C and D provide supplementary details for understanding various expressions in the paper. 


\section{Singlet fermion dark matter model}

We extend the SM by adding a new real scalar singlet $S$ and a Dirac fermion DM field $\psi$. The fermion DM is assumed to be living in the hidden sector and communicates with the SM particles only via the new scalar $S$. The model Lagrangian is given by [62]

$$
\mathscr{L}=\mathscr{L}_{\mathrm{SM}}+\mathscr{L}_{S}+\mathscr{L}_{\psi}+\mathscr{L}_{\text {portal }}
$$

where $\mathscr{L}_{\mathrm{SM}}$ is the SM Lagrangian,

$$
\begin{aligned}
\mathscr{L}_{S} & =\frac{1}{2}\left(\partial_{\mu} S\right)\left(\partial^{\mu} S\right)+\frac{1}{2} \mu_{S}^{2} S^{2}+\frac{1}{3} \mu_{3} S^{3}-\frac{1}{4} \lambda_{S} S^{4}, \\
\mathscr{L}_{\psi} & =\bar{\psi}\left(i \not \partial-\mu_{\psi}\right) \psi-g_{S} \bar{\psi} \psi S, \\
\mathscr{L}_{\text {portal }} & =-\mu_{\Phi S} \Phi^{\dagger} \Phi S-\frac{1}{2} \lambda_{\Phi S} \Phi^{\dagger} \Phi S^{2} .
\end{aligned}
$$

In general, a linear term in the $S$ field is allowed by symmetry. However, such a term can be removed by performing a constant shift in $S$ which also redefines $\mu_{S}^{2}, \mu_{\Phi}^{2}, \mu_{3}, g_{S}$ and $\mu_{\Phi S} .{ }^{1}$ In writing the above Lagrangians, we have assumed that these parameters are defined after a constant shift in $S$. If we set $\mu_{3}=g_{S}=\mu_{\Phi S}=0$, we can see that the above Lagrangian becomes $\mathbb{Z}_{2}$ symmetric under $S \rightarrow-S$, i.e., it is even in $S$. In this case, the fermion DM $\psi$ is decoupled and becomes a hidden DM candidate, whereas the scalar $S$ serves as a new DM candidate and reproduces the scalar Higgs portal model [36, 72-81].

With an extra scalar field, the tree-level scalar potential is given by

$$
V_{\text {tree }}=V_{\mathrm{SM}}+V_{S}+V_{\text {portal }}
$$

where $V_{S}$ and $V_{\text {portal }}$ can be read directly from eqs. (2.2) and (2.4) respectively. The SM part of the potential reads

$$
V_{\mathrm{SM}}=-\mu_{\Phi}^{2} \Phi^{\dagger} \Phi+\lambda_{\Phi}\left(\Phi^{\dagger} \Phi\right)^{2}
$$

where

$$
\Phi=\left(\begin{array}{c}
G^{+} \\
\frac{1}{\sqrt{2}}\left(\phi+i G^{0}\right)
\end{array}\right)
$$

is the SM Higgs doublet and $\left(G^{ \pm}, G^{0}\right)$ are the Goldstone bosons.

In general, both $\phi$ and $S$ can develop non-trivial vacuum expectation values (VEVs). At $T=0$, these are denoted by $v_{0}$ and $s_{0}$ respectively, i.e.,

$$
\left.\left.\langle 0|\phi| 0\rangle\right|_{T=0} \equiv\langle\phi\rangle\right|_{T=0}=v_{0},\left.\left.\quad\langle 0|S| 0\rangle\right|_{T=0} \equiv\langle S\rangle\right|_{T=0}=s_{0}
$$

After EWSB, we can expand $\Phi$ and $S$ in the unitary gauge as

$$
\Phi=\frac{1}{\sqrt{2}}\left(\begin{array}{c}
0 \\
v_{0}+\varphi
\end{array}\right), \quad S=s_{0}+s,
$$

\footnotetext{
${ }^{1}$ The parameter $\mu_{\Phi}^{2}$ appears in the SM Higgs potential, see eq. (2.6).
} 
where $(\varphi, s)$ fields represent quantum fluctuations around the $T=0 \mathrm{VEVs}$. Using the results presented in appendix A, we arrive at the following EWSB conditions

$$
\begin{aligned}
& \mu_{\Phi}^{2}=\lambda_{\Phi} v_{0}^{2}+\mu_{\Phi S} s_{0}+\frac{1}{2} \lambda_{\Phi S} s_{0}^{2}, \\
& \mu_{S}^{2}=-\mu_{3} s_{0}+\lambda_{S} s_{0}^{2}+\frac{\mu_{\Phi S} v_{0}^{2}}{2 s_{0}}+\frac{1}{2} \lambda_{\Phi S} v_{0}^{2} .
\end{aligned}
$$

The portal interaction Lagrangian in eq. (2.4) induces a mixing between the $\varphi$ and $s$ fields. Thus, the squared mass matrix

$$
\mathcal{M}^{2}=\left(\begin{array}{ll}
\mathcal{M}_{\varphi \varphi}^{2} & \mathcal{M}_{\varphi s}^{2} \\
\mathcal{M}_{s \varphi}^{2} & \mathcal{M}_{s s}^{2}
\end{array}\right)
$$

is non-diagonal. As shown in appendix A, its elements are given by

$$
\mathcal{M}_{\varphi \varphi}^{2}=2 \lambda_{\Phi} v_{0}^{2}, \quad \mathcal{M}_{s s}^{2}=-\mu_{3} s_{0}+2 \lambda_{S} s_{0}^{2}-\frac{\mu_{\Phi S} v_{0}^{2}}{2 s_{0}}, \quad \mathcal{M}_{\varphi s}^{2}=\mathcal{M}_{s \varphi}^{2}=\mu_{\Phi S} v_{0}+\lambda_{\Phi S} v_{0} s_{0}
$$

The squared mass matrix in eq. (2.12) can be diagonalised by rotating the interaction eigenstates $(\varphi, s)$ into the physical mass eigenstates $(h, H)$ as

$$
\left(\begin{array}{l}
h \\
H
\end{array}\right)=\left(\begin{array}{cc}
\cos \alpha & -\sin \alpha \\
\sin \alpha & \cos \alpha
\end{array}\right)\left(\begin{array}{l}
\varphi \\
s
\end{array}\right),
$$

where $\alpha$ is the mixing angle. Thus, for small mixing, $h$ is a SM-like Higgs boson, whereas $H$ is dominated by the scalar singlet.

For the tree-level scalar potential in eq. (2.5) to be bounded from below, the following conditions must be satisfied (see appendix A)

$$
\lambda_{\Phi}>0, \quad \lambda_{S}>0, \quad \lambda_{\Phi S}>-2 \sqrt{\lambda_{\Phi} \lambda_{S}} .
$$

After EWSB, the fermion DM Lagrangian in eq. (2.3) becomes

$$
\mathscr{L}_{\psi}=\bar{\psi}\left(i \not \partial-m_{\psi}\right) \psi-g_{S} \bar{\psi} \psi s,
$$

where

$$
m_{\psi}=\mu_{\psi}+g_{S} s_{0}
$$

is the physical fermion DM mass.

\section{Constraints and likelihoods}

In light of the recent discovery of a SM-like Higgs boson at the LHC [1, 2], we set

$$
m_{h}=125.13 \mathrm{GeV}, \quad v_{0}=246.22 \mathrm{GeV} .
$$

Thus, the model is completely described by the following 7 free parameters

$$
m_{H}, \quad s_{0}, \quad \mu_{3}, \quad \lambda_{S}, \quad \alpha, \quad m_{\psi}, \quad g_{S} .
$$


The remaining parameters in eqs. (2.2), (2.4) and (2.6) can be expressed as (see appendix B)

$$
\begin{aligned}
\lambda_{\Phi} & =\frac{1}{2 v_{0}^{2}}\left(m_{h}^{2} \cos ^{2} \alpha+m_{H}^{2} \sin ^{2} \alpha\right), \\
\mu_{\Phi S} & =-\frac{2 s_{0}}{v_{0}^{2}}\left(m_{h}^{2} \sin ^{2} \alpha+m_{H}^{2} \cos ^{2} \alpha+\mu_{3} s_{0}-2 \lambda_{S} s_{0}^{2}\right), \\
\lambda_{\Phi S} & =\frac{1}{v_{0} s_{0}}\left[\left(m_{H}^{2}-m_{h}^{2}\right) \sin \alpha \cos \alpha-\mu_{\Phi S} v_{0}\right], \\
\mu_{\Phi}^{2} & =\lambda_{\Phi} v_{0}^{2}+\mu_{\Phi S} s_{0}+\frac{1}{2} \lambda_{\Phi S} s_{0}^{2}, \\
\mu_{S}^{2} & =-\mu_{3} s_{0}+\lambda_{S} s_{0}^{2}+\frac{\mu_{\Phi S} v_{0}^{2}}{2 s_{0}}+\frac{1}{2} \lambda_{\Phi S} v_{0}^{2} .
\end{aligned}
$$

To study the phenomenology of our model, we implement the extended scalar singlet and fermion DM model in the LanHEP_v3.2.0 [82] package. For the calculation of the fermion DM relic density and Higgs decay rates, we use micrOMEGAs_v4.3.5 [83] which relies on the CalcHEP [84] package.

We make parameter inferences by adopting a frequentist approach and performing 7dimensional scans of the model parameter space using the Diver_v1.0.4 [85] package. ${ }^{2}$ The combined log-likelihood used in our global fit is

$$
\begin{aligned}
\ln \mathcal{L}_{\text {total }}(\boldsymbol{\theta})= & \ln \mathcal{L}_{\Omega h^{2}}(\boldsymbol{\theta})+\ln \mathcal{L}_{\mathrm{XENON} 1 \mathrm{~T}}(\boldsymbol{\theta})+\ln \mathcal{L}_{v_{c} / T_{c}}(\boldsymbol{\theta}) \\
& +\ln \mathcal{L}_{\mathrm{EWPO}}(\boldsymbol{\theta})+\ln \mathcal{L}_{\mathrm{HB}}(\boldsymbol{\theta})+\ln \mathcal{L}_{\mathrm{HS}}(\boldsymbol{\theta})
\end{aligned}
$$

where

- $\ln \mathcal{L}_{\Omega h^{2}}(\boldsymbol{\theta})$ : log-likelihood for the Planck measured DM relic density, see subsection 3.1 ;

- $\ln \mathcal{L}_{\text {XENON1T }}(\boldsymbol{\theta})$ : log-likelihood for the direct detection limits from the XENON1T (2018) experiment, see subsection 3.2;

- $\ln \mathcal{L}_{v_{c} / T_{c}}(\boldsymbol{\theta}): \log$-likelihood for the EWBG constraint, see subsection 3.3;

- $\ln \mathcal{L}_{\mathrm{EWPO}}(\boldsymbol{\theta})$ : log-likelihood for the electroweak precision observables (EWPO) constraint, see subsection 3.4;

- $\ln \mathcal{L}_{\mathrm{HB}}(\boldsymbol{\theta}): \log$-likelihood for the direct Higgs searches performed at the LEP, Tevatron and the LHC, see subsection 3.5;

- $\ln \mathcal{L}_{\mathrm{HS}}(\boldsymbol{\theta})$ : log-likelihood for the Higgs signal strength and mass measurements performed at the LHC, see subsection 3.5.

Here $\boldsymbol{\theta} \equiv\left(m_{H}, s_{0}, \mu_{3}, \lambda_{S}, \alpha, m_{\psi}, g_{S}\right)$ denotes the free parameters of our model. These are uniformly sampled over their ranges shown in table 1 in either flat or logarithmic space.

In the following subsections, we outline the details of all constraints and likelihoods used in our global fit.

\footnotetext{
${ }^{2}$ http://diver.hepforge.org.
} 


\begin{tabular}{|c|ccc|}
\hline Parameter & Minimum & Maximum & Prior type \\
\hline$m_{H}$ & $10 \mathrm{GeV}$ & $10 \mathrm{TeV}$ & log \\
$s_{0}$ & $-1 \mathrm{TeV}$ & $1 \mathrm{TeV}$ & flat \\
$\mu_{3}$ & $-1 \mathrm{TeV}$ & $1 \mathrm{TeV}$ & flat \\
$\lambda_{S}$ & $10^{-3}$ & 10 & log \\
$\alpha$ & 0 & $\pi$ & flat \\
$m_{\psi}$ & $10 \mathrm{GeV}$ & $10 \mathrm{TeV}$ & log \\
$g_{S}$ & $10^{-3}$ & 10 & log \\
\hline
\end{tabular}

Table 1. Ranges and priors for the free parameters of our model. All parameters are uniformly sampled over their ranges in either flat or logarithmic space. For the mixing angle $\alpha$, all constraints are symmetric under $\alpha \rightarrow-\alpha$, thus we only scan over $\alpha \in[0, \pi]$.

\subsection{Thermal relic density}

From the Planck satellite's observation of the temperature and polarization anisotropies in the cosmic microwave background (CMB), a strong bound on the present-day abundance of the DM particles can be extracted. The latest results indicate [67]

$$
\Omega_{\mathrm{DM}} h^{2}=0.1188 \pm 0.0010,
$$

where $\Omega_{\mathrm{DM}}=\rho_{\mathrm{DM}} / \rho_{c}$ is the density parameter, $\rho_{c}=3 H_{0}^{2} M_{p}^{2}$ is the critical mass density and $h=H_{0} /\left(100 \mathrm{~km} \mathrm{~s}^{-1} \mathrm{Mpc}^{-1}\right)$ is the reduced Hubble constant.

In our model, the Dirac fermion $\psi$ is the DM candidate. Its relic density is mainly determined by an $s$-channel annihilation into SM particles via an $h / H$ exchange. Annihilation into $h h, H H$ and $h H$ final states are also possible via the $t$ - and $u$-channels. Due to a mixing between the interaction eigenstates $(\varphi, s)$, the decay rates go as

$$
\begin{aligned}
\Gamma(h \rightarrow \bar{\psi} \psi) & \propto g_{S}^{2} \sin ^{2} \alpha, & \Gamma(h \rightarrow \overline{\mathcal{X}}) & \propto \cos ^{2} \alpha, \\
\Gamma(H \rightarrow \bar{\psi} \psi) & \propto g_{S}^{2} \cos ^{2} \alpha, & \Gamma(H \rightarrow \overline{\mathcal{X}}) & \propto \sin ^{2} \alpha,
\end{aligned}
$$

where $\mathcal{X}$ is a general SM final state, e.g., quarks, leptons or gauge bosons. Depending on the mixing angle $\alpha$, various SM and non-SM final states are allowed in the $s, t$ and $u$ channels.

1. $\alpha=0$ : in this case, $h$ is a SM-like Higgs boson, whereas $H$ is a scalar singlet. Thus, the only allowed final states from the fermion DM annihilation are $h h, H H$ and $h H$ via an $s$-channel $H$ exchange.

2. $\alpha=\pi / 2$ : in this case, $h$ is a scalar singlet, whereas $H$ is a SM-like Higgs boson. Similar to the $\alpha=0$ case, the only allowed final states from the fermion DM annihilation are $h h, H H$ and $h H$ via an $s$-channel $h$ exchange.

3. $\alpha \neq 0, \pi / 2$ : in these cases, all final states shown in figure 1 are allowed via either an $h$ or $H$ exchange. 

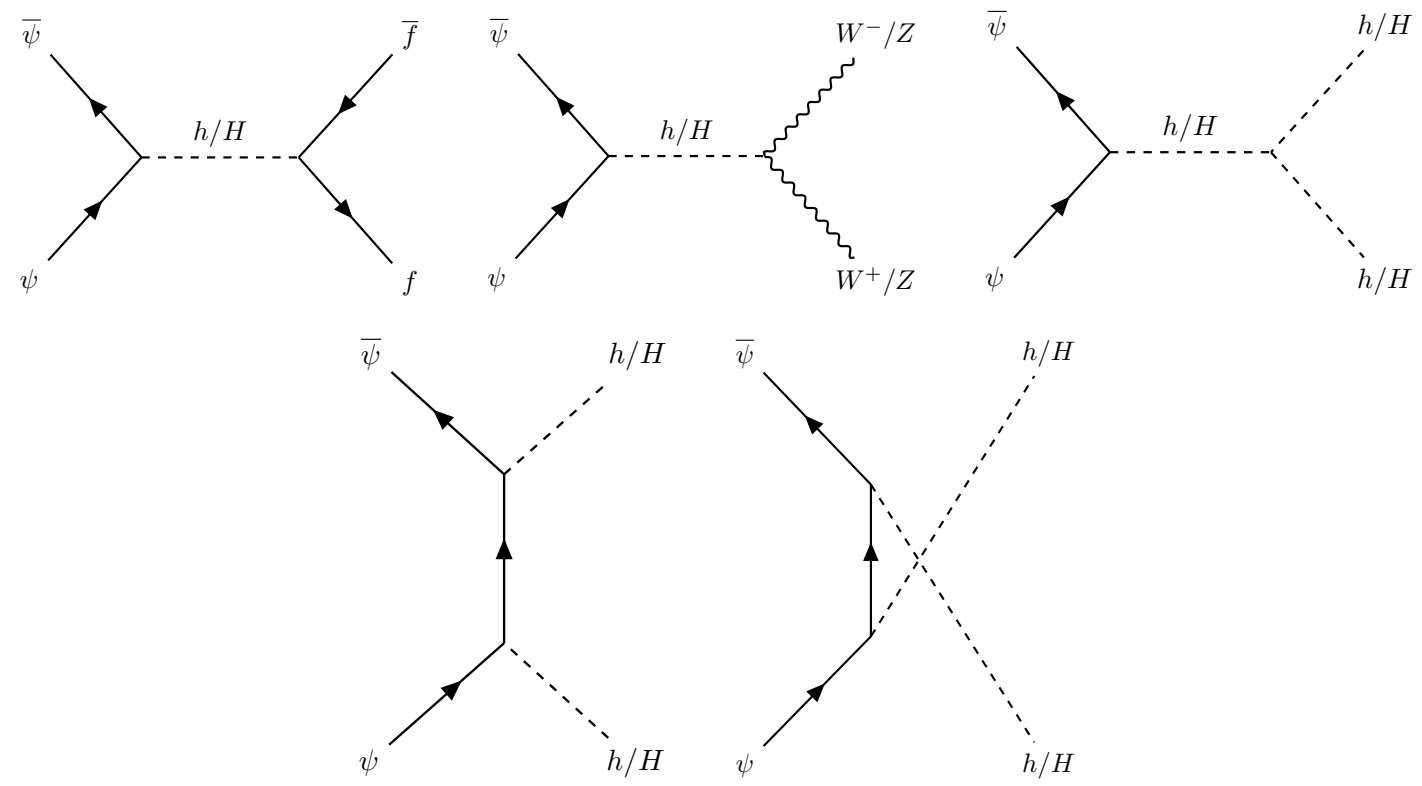

Figure 1. Feynman diagrams for the fermion DM annihilation into SM and $h / H$ particles when $\alpha \neq 0, \pi / 2$. Here $f$ refers to a SM fermion.

With two scalar mediators $h$ and $H$, the annihilation rate of the fermion DM into SM particles is enhanced when $m_{\psi} \sim m_{h, H} / 2$. At these two resonances, the fermion DM relic density $\Omega_{\psi} h^{2}$ drops rapidly with increasing scalar-fermion DM coupling $g_{S}$. For the fermion DM to account for the observed DM abundance, i.e., $\Omega_{\psi} h^{2}=\Omega_{\mathrm{DM}} h^{2}$, smaller values of $g_{S}$ are required to compensate for the enhanced DM annihilation rate into SM particles.

In order to address the strong possibility of a multicomponent dark sector, we define a relic abundance parameter $[72,73,86]$ as

$$
f_{\mathrm{rel}}=\frac{\Omega_{\psi}}{\Omega_{\mathrm{DM}}}
$$

where $\Omega_{\mathrm{DM}} h^{2}=0.1188$ is the Planck measured central value in eq. (3.9). Consequently, the indirect and direct detection rates must be scaled by $f_{\text {rel }}^{2}$ and $f_{\text {rel }}$ respectively. ${ }^{3}$ In regions of the model parameter space where $f_{\text {rel }}>1$, parameter points are robustly excluded by the relic density constraint.

We investigate both possibilities of our model to either account for all or part of the observed DM abundance. In the former case, we use a Gaussian likelihood function with a central value equal to the Planck measured one and a combined uncertainty equal to the Planck measured uncertainty with a $5 \%$ theoretical error. ${ }^{4}$ In the latter case, we instead

\footnotetext{
${ }^{3}$ In our study, we do not include any indirect detection limits as the fermion DM annihilation rate into $\mathrm{SM}$ particles is $p$-wave suppressed [87]. However, when a pure pseudoscalar, parity-violating interaction term $\left(\propto \bar{\psi} i \gamma_{5} \psi\right)$ is introduced, the resulting indirect detection limits can be sizeable [88-95].

${ }^{4} \mathrm{~A}$ possible source of theoretical uncertainty is in our relic density calculations as performed in micrOMEGAs.
} 
use a Gaussian likelihood function as an upper limit and require the parameter points to satisfy $f_{\text {rel }} \leq 1$. The results for both of these scenarios will be discussed in more detail in section 4 .

\subsection{Direct detection}

Direct detection experiments aim to measure the recoil of a nucleus from an elastic scattering off a DM particle. Such an event generates a typical recoil energy $E_{R}$ on the order of a few keV. As most radioactive elements and high-energy cosmic rays induce nuclear recoils with energies well above this value, direct DM searches must be conducted in deep underground laboratories to shield them from potential background sources.

In our model, the DM-quark interaction proceeds via a $t$-channel exchange of $h / H$ particles. With two neutral scalar mediators $(h, H)$, the resulting DM-nucleus interaction is nuclear spin-independent (SI). The SI DM-nucleus cross-section is given by

$$
\sigma_{\mathrm{SI}}^{\psi N}=\frac{\mu_{\psi N}^{2}}{\pi}\left[Z G_{p}+(A-Z) G_{n}\right]^{2}
$$

where $\mu_{\psi N}=m_{\psi} m_{N} /\left(m_{\psi}+m_{N}\right)$ is the DM-nucleus reduced mass and $Z(A-Z)$ are the number of protons (neutrons) in the target nucleus $N$. The dimensionful parameters $\left(G_{p}, G_{n}\right)$ are the effective DM-nucleon couplings. These are given by (see appendix C)

$$
G_{\mathcal{N}}=\frac{g_{S} \sin \alpha \cos \alpha}{v_{0}}\left(\frac{1}{m_{h}^{2}}-\frac{1}{m_{H}^{2}}\right) m_{\mathcal{N}} f_{\mathcal{N}}
$$

where $\mathcal{N} \in(p, n)$,

$$
f_{\mathcal{N}}=\frac{2}{9}+\frac{7}{9} \sum_{q=u, d, s} f_{T q}^{(\mathcal{N})}
$$

is the Higgs-nucleon coupling and

$$
f_{T q}^{(\mathcal{N})} \equiv \frac{m_{q}}{m_{\mathcal{N}}}\langle\mathcal{N}|\bar{q} q| \mathcal{N}\rangle
$$

are the hadronic matrix elements.

For isospin conserving couplings $\left(G_{p} \simeq G_{n}\right)$, the DM-nucleus cross-section in eq. (3.13) is enhanced by a factor of $A^{2}$. This is expected as the matrix element for a SI interaction involves a coherent sum over the individual protons and neutrons in the target nucleus $N$. For this reason, direct detection experiments rely on heavy target materials with large $Z$ to better constrain the DM-nucleon cross-section $\sigma_{\mathrm{SI}}^{\psi \mathcal{N}}$. In our model, it is given by

$$
\sigma_{\mathrm{SI}}^{\psi \mathcal{N}}=\frac{\mu_{\psi \mathcal{N}}^{2}}{\pi}\left(\frac{g_{S} \sin \alpha \cos \alpha}{v_{0}}\right)^{2}\left(\frac{1}{m_{h}^{2}}-\frac{1}{m_{H}^{2}}\right)^{2} m_{\mathcal{N}}^{2} f_{\mathcal{N}}^{2},
$$

where $\mu_{\psi \mathcal{N}}=m_{\psi} m_{\mathcal{N}} /\left(m_{\psi}+m_{\mathcal{N}}\right)$ is the DM-nucleon reduced mass, $m_{\mathcal{N}}=939 \mathrm{MeV}$ and $f_{\mathcal{N}}=0.3[72]$. 
Currently, the best upper limits on the SI DM-nucleon cross-section comes from the XENON1T (2018) experiment [68]. To constrain the model parameter space from the XENON1T experiment, we use a one-sided Gaussian likelihood function, i.e., we require the parameter points to satisfy ${ }^{5}$

$$
\sigma_{\mathrm{SI}}^{\mathrm{eff}} \leq \sigma_{\mathrm{XENON} 1 \mathrm{~T}}
$$

where $\sigma_{\text {XENON1T }}$ is the $90 \%$ C.L. upper limit from the XENON1T experiment and

$$
\sigma_{\mathrm{SI}}^{\mathrm{eff}}= \begin{cases}\sigma_{\mathrm{SI}}^{\psi \mathcal{N}} f_{\mathrm{rel}}, & f_{\text {rel }}<1, \\ \sigma_{\mathrm{SI}}^{\psi \mathcal{N}}, & f_{\text {rel }} \geq 1,\end{cases}
$$

is the effective SI DM-nucleon cross-section. The scaling of $\sigma_{\mathrm{SI}}^{\psi \mathcal{N}}$ by $f_{\text {rel }}$ is done to suppress signals when $f_{\text {rel }}<1$. In regions of the model parameter space where $f_{\text {rel }}>1$, parameter points are already ruled out by the relic density constraint.

We also include a theoretical uncertainty of $5 \%$ in our analysis. This can easily arise from the uncertainties associated with the nuclear physics, DM halo and velocity distribution parameters. For a recent review, see ref. [96].

\subsection{Electroweak baryogenesis (EWBG)}

In our model, the VEV of the new scalar $S$ does not initially have to be zero. Thus, the transition pattern can be $(\langle\phi\rangle,\langle s\rangle)=\left(0, s_{i}\right) \rightarrow(v, s)$. At low temperatures, the latter minimum evolves slowly to become the electroweak minimum at $T=0$, i.e., $(\langle\phi\rangle,\langle s\rangle)=\left(v_{0}, s_{0}\right)$. The initial transition can break the electroweak symmetry by tunnelling through a potential barrier to the broken phase minimum. This transition can proceed via nucleation of bubbles of the broken phase which results in a departure from thermal equilibrium [14-17]. In addition, it can generate a significant gravitational wave (GW) signal [97].

Using the standard notation, we define a strong first-order phase transition by

$$
\frac{v}{T} \gtrsim 1
$$

where $v$ is the Higgs VEV at temperature $T$. However, one has to keep in mind that the calculation of the baryon asymmetry remaining after the transition is quite complicated. This leads to a slightly different exact lower bound on $v / T$ [20,98-101].

To find regions in the model parameter space where a successful EWBG is potentially viable, we first find the minima of the effective potential $V_{\text {eff }}(\phi, S, T)$ (see appendix D) numerically, and compute the critical temperature $T_{c}$ at which the initial and symmetry breaking minima are degenerate. This allows us to compute the dimensionless parameter $v_{c} / T_{c}$ (the Higgs VEV $v_{c}$ at the critical temperature $T_{c}$ ) and constrain parts of the 7 dimensional model parameter space, i.e., parameter points are excluded if they lead to a

\footnotetext{
${ }^{5}$ The official XENON1T (2018) limits are only available for DM masses up to $1 \mathrm{TeV}$. Beyond $1 \mathrm{TeV}$, we perform a linear extrapolation of the limit due to the reduced DM number density.
} 
too weak phase transition. Specifically, we use a one-sided Gaussian likelihood function and require the parameter points to satisfy

$$
\frac{v_{c}}{T_{c}} \geq 0.6
$$

as a conservative limit. A theoretical uncertainity of $5 \%$ on the resulting $v_{c} / T_{c}$ values is assumed to obtain a smooth likelihood function. The actual uncertainty can be much larger as the value of $v_{c} / T_{c}$ required to facilitate EWBG is not yet settled.

In addition, parameter points are also excluded if they exhibit any of the following three features.

1. Incorrect minimum at $T=0$ : this situation arises when the electroweak vacuum $(\langle\phi\rangle,\langle S\rangle)=\left(v_{0}, s_{0}\right)$ is not the true minimum of the potential at $T=0$.

2. Runaway directions in the potential: this occur when the $\phi$ and $S$ field values in the symmetric or broken phase are too large, or if the potential in eq. (2.5) is unbounded from below in the general $\phi$ and $S$ directions, i.e., when $\lambda_{\Phi S} \leq-2 \sqrt{\lambda_{\Phi} \lambda_{S}}$.

3. Non-perturbative couplings: this situation arises when $\left|\lambda_{\Phi}\right|,\left|\lambda_{\Phi S}\right| \geq 4 \pi$. In this case, our 1-loop treatment of the effective potential is not reliable.

We also perform a complete analysis of the phase transition in this model by following our previous work on the $\mathbb{Z}_{2}$ symmetric case, i.e., scalar Higgs portal [36] and a very recent update on the calculation of the phase transition dynamics [102]. In particular, we find the percolation temperature $T_{p}$ at which the phase transition truly completes. This is used to compute the GW signals arising from the phase transition, and discuss the potential discovery prospects of the model at current and future GW experiments. For more details, see section 4 .

Let us also point out that we only check one of the necessary conditions for a successful EWBG, while other difficulties might still arise. For instance, the standard mechanism of generating a baryon yield requires a sufficiently slow speed of the expanding bubble walls [103-105]. We do not compute the bubble wall velocity to check this requirement (in fact, we assume it to be very high) while calculating the GW spectra. While there are mechanisms which could generate the asymmetry even for very fast walls [106-108], we also do not explicitly make sure that other conditions they carry are fulfilled.

\subsection{Electroweak precision observables (EWPO)}

With an extra scalar, our model can induce corrections to the gauge boson self-energy diagrams. Its effect on the electroweak precision observables (EWPO) can be parametrised by the oblique parameters $S, T$ and $U$ [109]. The $\gamma \gamma$ and $\gamma Z$ self-energies $\left(\Pi_{\gamma \gamma}\right.$ and $\Pi_{\gamma Z}$ respectively) are not modified as the new scalar is electrically neutral. Thus, only the $W$ and $Z$ boson self-energies are subject to corrections. 
In our model, the oblique parameters are shifted from their SM values by [62]

$$
\begin{aligned}
\Delta T= & \frac{3}{16 \pi s_{W}^{2}}\left[\cos ^{2} \alpha\left\{f_{T}\left(\frac{m_{h}^{2}}{m_{W}^{2}}\right)-\frac{1}{c_{W}^{2}} f_{T}\left(\frac{m_{h}^{2}}{m_{Z}^{2}}\right)\right\}+\sin ^{2} \alpha\left\{f_{T}\left(\frac{m_{H}^{2}}{m_{W}^{2}}\right)\right.\right. \\
& \left.\left.-\frac{1}{c_{W}^{2}} f_{T}\left(\frac{m_{H}^{2}}{m_{Z}^{2}}\right)\right\}-\left\{f_{T}\left(\frac{m_{h}^{2}}{m_{W}^{2}}\right)-\frac{1}{c_{W}^{2}} f_{T}\left(\frac{m_{h}^{2}}{m_{Z}^{2}}\right)\right\}\right], \\
\Delta S= & \frac{1}{2 \pi}\left[\cos ^{2} \alpha f_{S}\left(\frac{m_{h}^{2}}{m_{Z}^{2}}\right)+\sin ^{2} \alpha f_{S}\left(\frac{m_{H}^{2}}{m_{Z}^{2}}\right)-f_{S}\left(\frac{m_{h}^{2}}{m_{Z}^{2}}\right)\right], \\
\Delta U= & \frac{1}{2 \pi}\left[\cos ^{2} \alpha f_{S}\left(\frac{m_{h}^{2}}{m_{W}^{2}}\right)+\sin ^{2} \alpha f_{S}\left(\frac{m_{H}^{2}}{m_{W}^{2}}\right)-f_{S}\left(\frac{m_{h}^{2}}{m_{W}^{2}}\right)\right]-\Delta S,
\end{aligned}
$$

where $\Delta \mathcal{O} \equiv \mathcal{O}-\mathcal{O}_{\mathrm{SM}}$ for $\mathcal{O} \in(S, T, U), m_{W}\left(m_{Z}\right)$ is the $W(Z)$ boson mass, $c_{W}^{2}=m_{W}^{2} / m_{Z}^{2}$ and $s_{W}^{2}=1-c_{W}^{2}$. The loop functions $f_{T}(x)$ and $f_{S}(x)$ are given by [110]

$$
\begin{aligned}
& f_{T}(x)=\frac{x \log x}{x-1}, \\
& f_{S}(x)=\left\{\begin{array}{l}
\frac{1}{12}\left[-2 x^{2}+9 x+\left((x-3)\left(x^{2}-4 x+12\right)+\frac{1-x}{x}\right) f_{T}(x)\right. \\
\left.+2 \sqrt{(4-x) x}\left(x^{2}-4 x+12\right) \tan ^{-1}\left(\sqrt{\frac{4-x}{x}}\right)\right], \quad 0<x<4, \\
\frac{1}{12}\left[-2 x^{2}+9 x+\left((x-3)\left(x^{2}-4 x+12\right)+\frac{1-x}{x}\right) f_{T}(x)\right. \\
\left.+\sqrt{(x-4) x}\left(x^{2}-4 x+12\right) \log \left(\frac{x-\sqrt{(x-4) x}}{x+\sqrt{(x-4) x}}\right)\right], \quad x \geq 4 .
\end{array}\right.
\end{aligned}
$$

These are also plotted in figure 2. From eqs. (3.22)-(3.24), it is evident that

$$
\Delta \mathcal{O}=\left(1-\cos ^{2} \alpha\right)\left[\mathcal{O}_{\mathrm{SM}}\left(m_{H}\right)-\mathcal{O}_{\mathrm{SM}}\left(m_{h}\right)\right] .
$$

Thus, for large $m_{H}, \alpha \sim 0, \pi$ is required, whereas large mixing angles are compatible with the EWPO constraint provided $m_{H} \sim m_{h}$.

Using the SM reference as $m_{h}^{\text {ref }}=125 \mathrm{GeV}$ and $m_{t}^{\text {ref }}=172.5 \mathrm{GeV}$, the most recent global electroweak fit gives [69]

$$
\Delta S=0.04 \pm 0.11, \quad \Delta T=0.09 \pm 0.14, \quad \Delta U=-0.02 \pm 0.11
$$

and the following correlation matrix

$$
\rho_{i j}=\left(\begin{array}{ccc}
1 & 0.92 & -0.68 \\
0.92 & 1 & -0.87 \\
-0.68 & -0.87 & 1
\end{array}\right) .
$$

To constrain the model parameter space from the EWPO, we use the following likelihood function [111]

$$
\ln \mathcal{L}_{\mathrm{EWPO}}(\boldsymbol{\theta})=-\frac{1}{2} \Delta \chi^{2}=-\frac{1}{2} \sum_{i, j}\left(\Delta \mathcal{O}_{i}-\overline{\Delta \mathcal{O}}_{i}\right)\left(\Sigma^{2}\right)_{i j}^{-1}\left(\Delta \mathcal{O}_{j}-\overline{\Delta \mathcal{O}}_{j}\right),
$$




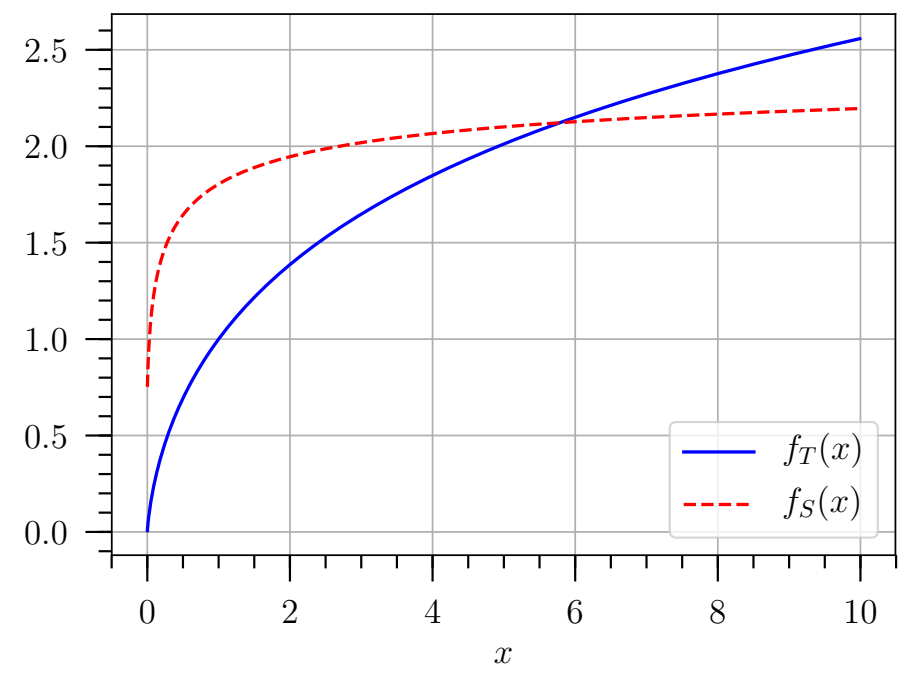

Figure 2. Loop functions $f_{T}(x)$ (solid blue) and $f_{S}(x)$ (dashed red).

where $\overline{\Delta \mathcal{O}}_{i}$ denotes the central values for the shifts in eq. (3.28), $\Sigma_{i j}^{2} \equiv \sigma_{i} \rho_{i j} \sigma_{j}$ is the covariance matrix, $\rho_{i j}$ is the correlation matrix in eq. (3.29) and $\sigma_{i}$ are the associated errors in eq. (3.28).

\subsection{Higgs searches at colliders}

Due to a mixing between the interaction eigenstates $(\varphi, s)$, the coupling strengths between the mass eigenstates $(h, H)$ and SM particles are modified with respect to the SM expectation. The effective squared couplings of $(h, H)$ to SM particles are [66]

$$
\left(\frac{g_{h \mathcal{X} \overline{\mathcal{X}}}}{g_{h \mathcal{X} \overline{\mathcal{X}}}^{\mathrm{SM}}}\right)^{2}=\cos ^{2} \alpha, \quad\left(\frac{g_{H \mathcal{X} \overline{\mathcal{X}}}}{g_{H \mathcal{X} \overline{\mathcal{X}}}^{\mathrm{SM}}}\right)^{2}=\sin ^{2} \alpha,
$$

where $\mathcal{X}$ refers to a SM quark, lepton or gauge boson, and $g_{h \mathcal{X} \mathcal{X}}^{\mathrm{SM}}\left(g_{H \mathcal{X} \overline{\mathcal{X}}}^{\mathrm{SM}}\right)$ are the coupling strengths for a SM-like Higgs boson with mass $m_{h}\left(m_{H}\right)$. For the loop-induced processes, the effective squared couplings are given by [112]

$$
\left(\frac{g_{h \mathcal{Y} \overline{\mathcal{Y}}}}{g_{h \mathcal{Y} \overline{\mathcal{Y}}}^{\mathrm{SM}}}\right)^{2}=\frac{\Gamma_{h \rightarrow \mathcal{Y} \overline{\mathcal{Y}}}}{\Gamma_{h \rightarrow \mathcal{Y} \overline{\mathcal{Y}}}^{\mathrm{SM}}}=\cos ^{2} \alpha, \quad\left(\frac{g_{H \mathcal{Y} \overline{\mathcal{Y}}}}{g_{H \mathcal{Y} \overline{\mathcal{Y}}}^{\mathrm{SM}}}\right)^{2}=\frac{\Gamma_{H \rightarrow \mathcal{Y} \overline{\mathcal{Y}}}}{\Gamma_{H \rightarrow \mathcal{Y} \overline{\mathcal{Y}}}^{\mathrm{SM}}}=\sin ^{2} \alpha,
$$

where $\mathcal{Y} \overline{\mathcal{Y}} \in(\gamma \gamma, \gamma Z, g g, g g Z)$ and $\Gamma_{h \rightarrow \mathcal{Y}}^{\mathrm{SM}}\left(\Gamma_{H \rightarrow \mathcal{Y}}^{\mathrm{SM}}\right)$ are the decay rates for a SM-like Higgs boson with mass $m_{h}\left(m_{H}\right)$. With modified branching ratios of $h / H$ into SM particles, the scalar sector of our model can be constrained using the direct Higgs searches performed at the lepton (e.g., LEP) and hadron (e.g., Tevatron, LHC) colliders.

To constrain the model parameter space from the direct Higgs searches performed at the LEP, Tevatron and the LHC, we use the HiggsBounds_v4.3.1 [70] package. From the model predictions for the two scalar masses, total decay widths, branching ratios, and effective squared couplings defined in eqs. (3.31) and (3.32), HiggsBounds computes 
and compares the predicted signal rates for the search channels considered in multiple experimental analyses. By comparing the predicted signal rates against the expected and observed cross-section limits from the direct Higgs searches, it determines whether or not a given parameter point is excluded at $95 \%$ C.L.

For the two physical scalars $(h, H)$, the signal strengths are given by [66]

$$
\begin{aligned}
\mu_{h} & =\frac{\Gamma_{h}^{\mathrm{SM}} \cos ^{4} \alpha}{\Gamma_{h}^{\mathrm{SM}} \cos ^{2} \alpha+\Gamma_{h \rightarrow \bar{\psi} \psi}+\Gamma_{h \rightarrow H H}}, \\
\mu_{H} & =\frac{\Gamma_{H}^{\mathrm{SM}} \sin ^{4} \alpha}{\Gamma_{H}^{\mathrm{SM}} \sin ^{2} \alpha+\Gamma_{H \rightarrow \bar{\psi} \psi}+\Gamma_{H \rightarrow h h}} .
\end{aligned}
$$

In the absence of invisible and cross Higgs decay modes, $\mu_{h}\left(\mu_{H}\right)$ scales as $\cos ^{2} \alpha\left(\sin ^{2} \alpha\right)$. However, when these decay modes are kinematically allowed, they suppress the $h / H$ signal strength with respect to the SM expectation. Thus, the scalar sector of our model can also be constrained using the Higgs signal strength and mass measurements performed at the LHC.

To constrain the model parameter space from the Higgs signal strength and mass measurements, we use the HiggsSignals_v1.4.0 [71] package. Assuming a Gaussian probability density function (p.d.f.) for the two scalar masses, we compute a chi-square $\chi_{\mathrm{HS}}^{2}$ using the peak-centered method. ${ }^{6}$ In this method, $\chi_{\mathrm{HS}}^{2}$ is evaluated by assigning, for each signal (or peak) observed in multiple experimental analyses (see table 2), a combination of the two Higgs bosons from our model provided their masses lie within the experimental resolution of an analysis [118]. Following the assignment, a $\chi_{\mu}^{2}$ is evaluated by comparing the signal strength measurement for the peak to the model predicted signal strength. When a mass measurement is also available (e.g., from channels with a good mass-resolution such as the $h \rightarrow \gamma \gamma$ decay mode), a corresponding $\chi_{m}^{2}$ is also evaluated by comparing the model predicted and observed Higgs boson mass. Thus, the total $\chi_{\mathrm{HS}}^{2}$ is given by ${ }^{7}$

$$
\chi_{\mathrm{HS}}^{2}=\chi_{\mu}^{2}+\chi_{m}^{2}=\chi_{\mu}^{2}+\sum_{i=1}^{2} \chi_{m_{i}}^{2} .
$$

In situations where more than one Higgs boson can contribute to a signal (as in our case), an optimal assignment of the Higgs bosons to the signals is achieved by minimising the overall $\chi_{\mathrm{HS}}^{2}$. The predicted signal strengths of the two scalars are added incoherently, assuming negligible interference effects. Finally, the computed $\chi_{\mathrm{HS}}^{2}$ is used to define a Higgs signal strength log-likelihood as

$$
\ln \mathcal{L}_{\mathrm{HS}}(\boldsymbol{\theta})=-\frac{1}{2} \chi_{\mathrm{HS}}^{2}
$$

Thus, a large $\chi_{\mathrm{HS}}^{2}$ indicates a large deviation between the model predicted signal strength and the best-fit value for a fixed Higgs boson mass, and vice versa.

\footnotetext{
${ }^{6} \mathrm{~A}$ theoretical mass uncertainty of zero is assumed for both scalars as $m_{h}$ is fixed, whereas $m_{H}$ is a free model parameter.

${ }^{7}$ For more details on the functional form of individual chi-squares, see ref. [71].
} 


\begin{tabular}{|c|ccc|}
\hline Experiment & Channel & Obs. signal strength & Ref. \\
\hline ATLAS & $h \rightarrow W W^{*}$ & $1.18_{-0.21}^{+0.24}$ & {$[113]$} \\
ATLAS & $h \rightarrow Z Z^{*}$ & $1.46_{-0.34}^{+0.40}$ & {$[113]$} \\
ATLAS & $h \rightarrow \gamma \gamma$ & $1.17_{-0.26}^{+0.28}$ & {$[113]$} \\
ATLAS & $h \rightarrow \tau^{+} \tau^{-}$ & $1.44_{-0.37}^{+0.42}$ & {$[113]$} \\
ATLAS & $h \rightarrow b \bar{b}$ & $0.63_{-0.37}^{+0.39}$ & {$[113]$} \\
CMS & $h \rightarrow W W^{*}$ & $0.72_{-0.18}^{+0.20}$ & {$[114]$} \\
CMS & $h \rightarrow Z Z^{*}$ & $0.93_{-0.25}^{+0.29}$ & {$[115]$} \\
CMS & $h \rightarrow \gamma \gamma$ & $1.14_{-0.23}^{+0.26}$ & {$[116]$} \\
CMS & $h \rightarrow \tau^{+} \tau^{-}$ & $0.78_{-0.27}^{+0.27}$ & {$[117]$} \\
CMS & $h \rightarrow b \bar{b}$ & $1.00_{-0.50}^{+0.50}$ & {$[117]$} \\
\hline
\end{tabular}

Table 2. A summary of Higgs boson signal strength measurements that are included in our analysis. For more details, see Expt_tables/latestresults-1.4.0-LHCinclusive/ directory of HiggsSignals_v1.4.0 [71].

\section{Results}

We perform scans of our 7D model parameter space using Diver_v1.0.4 [85] with lambdajDE $=$ true, $\mathrm{NP}=50,000$ and convthresh $=10^{-5}$. To efficiently sample all parts of the parameter space (even the degenerate ones), we also run several targeted scans and combine the output chains to obtain high-quality profile likelihood (PL) plots.

We present our model results in the form of 1- and 2-dimensional PL plots. For a model parameter $\theta_{i}$ where $i=1, \ldots, 7$, a $1 \mathrm{D} \mathrm{PL} \mathcal{L}_{\mathrm{PL}}\left(\theta_{i}\right)$ is defined as

$$
\mathcal{L}_{\mathrm{PL}}\left(\theta_{i}\right) \equiv \max _{\left\{\theta_{j} \mid j \neq i\right\}} \mathcal{L}(\boldsymbol{\theta})
$$

Thus, $\mathcal{L}_{\mathrm{PL}}\left(\theta_{i}\right)$ is a function of $\theta_{i}$ only, i.e., all other parameters are profiled out. Similarly, a $2 \mathrm{D} \operatorname{PL} \mathcal{L}_{\mathrm{PL}}\left(\theta_{i}, \theta_{j}\right)$ is defined as

$$
\mathcal{L}_{\mathrm{PL}}\left(\theta_{i}, \theta_{j}\right) \equiv \max _{\left\{\theta_{k} \mid k \neq i, j\right\}} \mathcal{L}(\boldsymbol{\theta})
$$

Thus, $\mathcal{L}_{\mathrm{PL}}\left(\theta_{i}, \theta_{j}\right)$ is a function of $\theta_{i}$ and $\theta_{j}$ only. Using eqs. (4.1) and (4.2), we can define a $\mathrm{PL}$ ratio $[119]$ as

$$
\Lambda\left(\theta_{i}\right)=\frac{\mathcal{L}_{\mathrm{PL}}\left(\theta_{i}\right)}{\mathcal{L}(\hat{\boldsymbol{\theta}})}, \quad \Lambda\left(\theta_{i}, \theta_{j}\right)=\frac{\mathcal{L}_{\mathrm{PL}}\left(\theta_{i}, \theta_{j}\right)}{\mathcal{L}(\hat{\boldsymbol{\theta}})}
$$

where $\hat{\boldsymbol{\theta}} \equiv\left(\hat{\theta}_{1}, \ldots, \hat{\theta}_{7}\right)$ is the best-fit point, i.e., a parameter point that maximises the total likelihood function $\mathcal{L}(\boldsymbol{\theta})$. Using Wilks' theorem [120], eq. (4.3) can be used to construct $1 \sigma(2 \sigma)$ contours corresponding to $\sim 68.3 \%$ (95.4\%) C.L. regions.

In the following subsections, we present our model results in the form of $1 \mathrm{D}$ and $2 \mathrm{D}$ PL plots. These are generated using the pippi_v2.0 [121] package. 


\subsection{EWBG only}

We start by finding regions in the model parameter space where a successful EWBG is potentially viable. This is achieved by performing a $7 \mathrm{D}$ scan of the model using only the $v_{c} / T_{c} \log$-likelihood, i.e.,

$$
\ln \mathcal{L}(\boldsymbol{\theta})=\ln \mathcal{L}_{v_{c} / T_{c}}(\boldsymbol{\theta}),
$$

where $\ln \mathcal{L}_{v_{c} / T_{c}}(\boldsymbol{\theta})$ is defined in subsection 3.3. The resulting 2D PL plots are shown in figure 3. In the dark blue regions where the PL ratio $\Lambda \equiv \mathcal{L} / \mathcal{L}_{\max }=1$, the dimensionless parameter $v_{c} / T_{c} \geq 0.6$ and a successful EWBG can be viable. To understand the results in more detail, we go over each panel in figure 3 one-by-one.

1. $\left(m_{H}, s_{0}\right)$ plane: for $m_{H} \lesssim 1.3 \mathrm{TeV}$, all values of $s_{0}$ and some combination of 5 profiled out parameters (namely $\mu_{3}, \lambda_{S}, \alpha, m_{\psi}$ and $g_{S}$ ) give $v_{c} / T_{c} \geq 0.6$ and maximise the $v_{c} / T_{c} \log$-likelihood, thus $\Lambda=1$ everywhere. Due to the dependence of $s_{0}$ in eq. (3.5), large values of $\left|s_{0}\right|$ should lead to runaway directions, $\lambda_{\Phi S} \leq-2 \sqrt{\lambda_{\Phi} \lambda_{S}}$, and/or nonperturbative coupling, $\left|\lambda_{\Phi S}\right| \geq 4 \pi$. With $\alpha=\pi / 2$, a large contribution from $m_{H}$ to $\lambda_{\Phi S}$ can be suppressed. However, this choice of $\alpha$ makes $\lambda_{\Phi}$ in eq. (3.3) nonperturbative as its contribution appears as $m_{H}^{2} \sin ^{2} \alpha$. Ultimately, the solution is to choose a small value for $\lambda_{S}$ as its contribution in eq. (3.5) appears as $-\lambda_{S} s_{0}^{2}$. In addition, small values of $\mu_{3}$ can also help in keeping $\left|\lambda_{\Phi S}\right|<4 \pi$. Thus, for $m_{H} \lesssim 1.3 \mathrm{TeV}$, large values of $\left|s_{0}\right|$ can facilitate EWBG.

For $m_{H} \gtrsim 1.3 \mathrm{TeV}$ and $\left|s_{0}\right| \gtrsim 50 \mathrm{GeV}$, the white region $(\Lambda=0)$ is disfavoured as it leads to $\left|\lambda_{\Phi S}\right| \geq 4 \pi$. This is expected as the contribution from $m_{H}$ in eq. (3.5) is dominant at large values. With large $\left|s_{0}\right|$, no choice of $\mu_{3}, \lambda_{S}$ and $\alpha$ can keep $\left|\lambda_{\Phi S}\right|<4 \pi$. In fact, the requirement $\left|\lambda_{\Phi S}\right|<4 \pi$ translates into an upper limit on $m_{H}$ as a function of $s_{0}, \mu_{3}, \lambda_{S}$ and $\alpha$. Using eq. (3.5), we get

$$
\frac{v_{0}}{s_{0}}\left(m_{H}^{2}-m_{h}^{2}\right) \sin 2 \alpha+4\left(m_{h}^{2} \sin ^{2} \alpha+m_{H}^{2} \cos ^{2} \alpha+\mu_{3} s_{0}-2 \lambda_{S} s_{0}^{2}\right)<8 \pi v_{0}^{2} .
$$

For a fixed $m_{H}$ and $s_{0}$, eq. (4.5) has 3 degrees of freedom. As $\mu_{3}, \lambda_{S}$ and $\alpha$ are profiled over, it is non-trivial to predict the exact shape of the upper limit on $m_{H}$ as a function of $s_{0}$. The upper limit also weakens as $\left|s_{0}\right|$ increases. The net result is that for $m_{H} \gtrsim 5 \mathrm{TeV},\left|s_{0}\right| \lesssim 50 \mathrm{GeV}$ is required to facilitate EWBG.

2. $\left(m_{H}, \alpha\right)$ plane: similar to the $\left(m_{H}, s_{0}\right)$ plane for $m_{H} \lesssim 1.3 \mathrm{TeV}$, some combination of the profiled out parameters gives $v_{c} / T_{c} \geq 0.6$ for all values of $\alpha$. However, when $m_{H} \gtrsim 1.3 \mathrm{TeV}$ and $\alpha \neq 0, \pi$, the Higgs quartic coupling $\lambda_{\Phi}$ in eq. (3.3) becomes non-perturbative. In fact, the requirement $\left|\lambda_{\Phi}\right|<4 \pi$ translates into the following upper limit on $m_{H}$ as a function of $\alpha$

$$
m_{H}^{2} \sin ^{2} \alpha<8 \pi v_{0}^{2}-m_{h}^{2} \cos ^{2} \alpha .
$$

When $\alpha=0, \pi$, the above condition is satisfied for all values of $m_{H}$. Thus, a successful EWBG can be viable at large values of $m_{H}$. On the other hand, when $\alpha=\pi / 2$, eq. (4.6) imposes the strongest upper limit on $m_{H}$, namely $m_{H} \lesssim 1.23 \mathrm{TeV}$. As $\alpha \rightarrow 0, \pi$, the upper limit on $m_{H}$ becomes weaker, as is evident from the plot. 

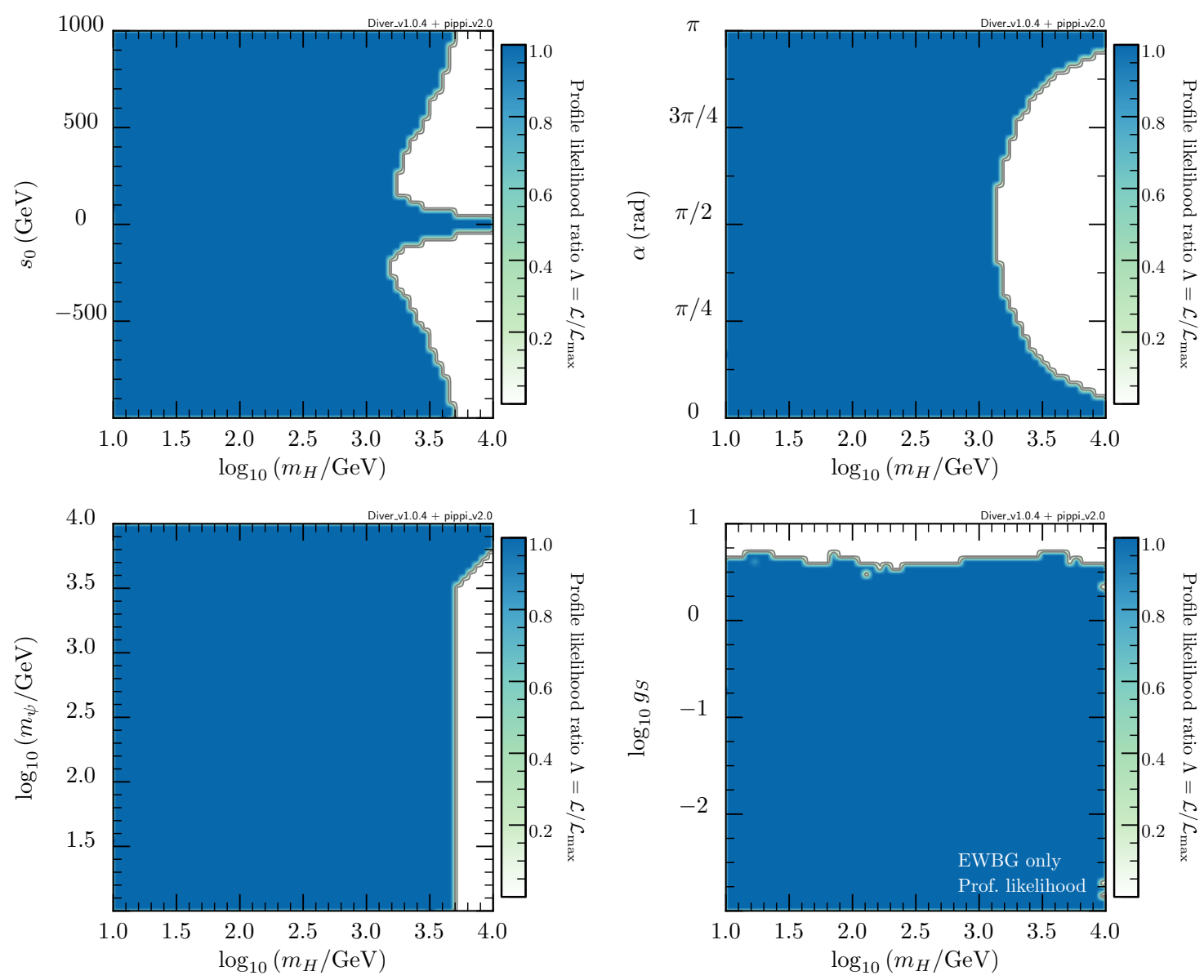

Figure 3. 2D profile likelihood (PL) plots from a 7D scan of our model using only the electroweak baryogenesis (EWBG) constraint. The contour lines mark out the $1 \sigma(68.3 \%)$ and $2 \sigma(95.4 \%)$ C.L. regions. In regions where $\Lambda \equiv \mathcal{L} / \mathcal{L}_{\text {max }}=1$, a successful EWBG can be viable as $v_{c} / T_{c} \geq 0.6$ (see text for more details). The parameter planes $\left(m_{H}, \mu_{3}\right)$ and $\left(m_{H}, \lambda_{S}\right)$ are not shown as they are unconstrained by the EWBG constraint.

3. $\left(m_{H}, \mu_{3}\right)$ and $\left(m_{H}, \lambda_{S}\right)$ planes: in these two planes, all possible combinations of $\left(m_{H}, \mu_{3}\right),\left(m_{H}, \lambda_{S}\right)$ and profiled out parameters give $v_{c} / T_{c} \geq 0.6$. Thus, the PL ratio is roughly flat and equal to 1 everywhere; hence, we do not show these planes in figure 3. In fact, the $v_{c} / T_{c}$ likelihood is weakly dependent on $\mu_{3}$ and $\lambda_{S}$ as expected from eq. (3.5). For instance, at large values of $\mu_{3}$ or $\lambda_{S}$ which would give $\left|\lambda_{\Phi S}\right| \geq 4 \pi$ or $\lambda_{\Phi S} \leq-2 \sqrt{\lambda_{\Phi} \lambda_{S}}$, small values of $s_{0}$ can be chosen to avoid such situations.

4. $\left(m_{H}, m_{\psi}\right)$ plane: for $m_{H} \lesssim 5 \mathrm{TeV}$, all values of $m_{\psi}$ give $v_{c} / T_{c} \geq 0.6$. As $m_{\psi}$ does not appear directly in eqs. (3.3) and (3.5), the $v_{c} / T_{c}$ likelihood is weakly dependent on $m_{\psi}$. This is expected as the contribution from $m_{\psi}$ to the effective potential appears only at 1-loop order.

For $m_{H} \gtrsim 5 \mathrm{TeV}$ and $m_{\psi} \lesssim 3.2 \mathrm{TeV}$, no combination of the profiled out parameters can keep $\left|\lambda_{\Phi S}\right|<4 \pi$. On the other hand, when $m_{\psi} \gtrsim 3.2 \mathrm{TeV}$, one can arrange for a 
cancellation of large quantum corrections to obtain perturbative couplings, although all such solutions carry some degree of extra tuning.

5. $\left(m_{H}, g_{S}\right)$ plane: for $g_{S} \lesssim 5.62$, all values of $m_{H}$ and profiled out parameters give $v_{c} / T_{c} \geq 0.6$, and maximise the $v_{c} / T_{c}$ likelihood. However, values of $g_{S}>5.62$ lead to runaway directions in the potential as the contribution from $g_{S}$ in the 1-loop corrections become large.

In summary, it is not difficult to facilitate a successful EWBG in our model. For any specific model parameter, usually some combination of the remaining parameters give viable points even if the parameter in question causes problems. For instance, large values of $m_{H}$ generally push up the EWSB minimum and cause it to not become the global minimum at $T=0$. However, this effect can be counteracted by choosing a large value for $m_{\psi}$ which gives a large negative contribution to the effective potential. One exception is $g_{S}>5.62$ which always generates runaway directions in the effective potential. For the remaining model parameters, namely $\left(m_{H}, s_{0}, \mu_{3}, \lambda_{S}, \alpha, m_{\psi}\right)$, the $1 \mathrm{D}$ PL ratio $\Lambda$ is roughly flat and equal to 1 for all parameter values. Thus, we do not show the 1D PL plots for our model parameters.

\subsection{Global fit}

With some intuition on the choice of free model parameters that can facilitate a successful EWBG, we present results from a global fit of our model using the total log-likelihood function in eq. (3.8). In practice, we consider two scenarios in which the fermion DM accounts for either a small fraction $\left(f_{\text {rel }} \leq 1\right)$ or all $\left(f_{\text {rel }}=1\right)$ of the observed DM abundance. In the former case, we use a relic density likelihood that is one-sided Gaussian, whereas in the latter, we use a Gaussian likelihood. For more details, see subsection 3.1.

\subsubsection{Scenario I: $f_{\text {rel }} \leq 1$}

The resulting 2D PL plots from our 7D scans are shown in figure 4 . For $m_{H} \lesssim m_{h} / 2=$ $62.6 \mathrm{GeV}$, the parameter planes are ruled out by the observed Higgs signal strength measurements, EWPO and direct Higgs searches performed at the LEP experiment. As the decay channel $h \rightarrow H H$ is kinematically allowed and dominant in this region for all values of the mixing angle $\alpha$, it reduces the SM-like Higgs signal strength $\mu_{h}$ with respect to SM expectation, see eq. (3.33). This translates into a large $\chi_{\mu}^{2}$ in eq. (3.36) and is thus disfavoured.

To understand the remaining set of results in more detail, we go over each panel in figure 4 one-by-one.

1. $\left(m_{H}, s_{0}\right)$ plane: for $m_{H} \gtrsim 4 \mathrm{TeV}$, the parameter planes are ruled out by the EWBG constraint as they either lead to runaway directions, $\lambda_{\Phi S} \leq-2 \sqrt{\lambda_{\Phi} \lambda_{S}}$, or nonperturbative couplings, $\left|\lambda_{\Phi}\right|,\left|\lambda_{\Phi S}\right| \geq 4 \pi$. Although, some combinations of the profiled out parameters can give a successful EWBG at large values of $m_{H}$ (see figure 3), they are often not compatible with the remaining constraints. This is especially true for the EWPO constraint which only depends on $m_{H}$ and $\alpha$. For large $m_{H}, \alpha \simeq 0, \pi$ is required in order to satisfy the EWPO constraint. 

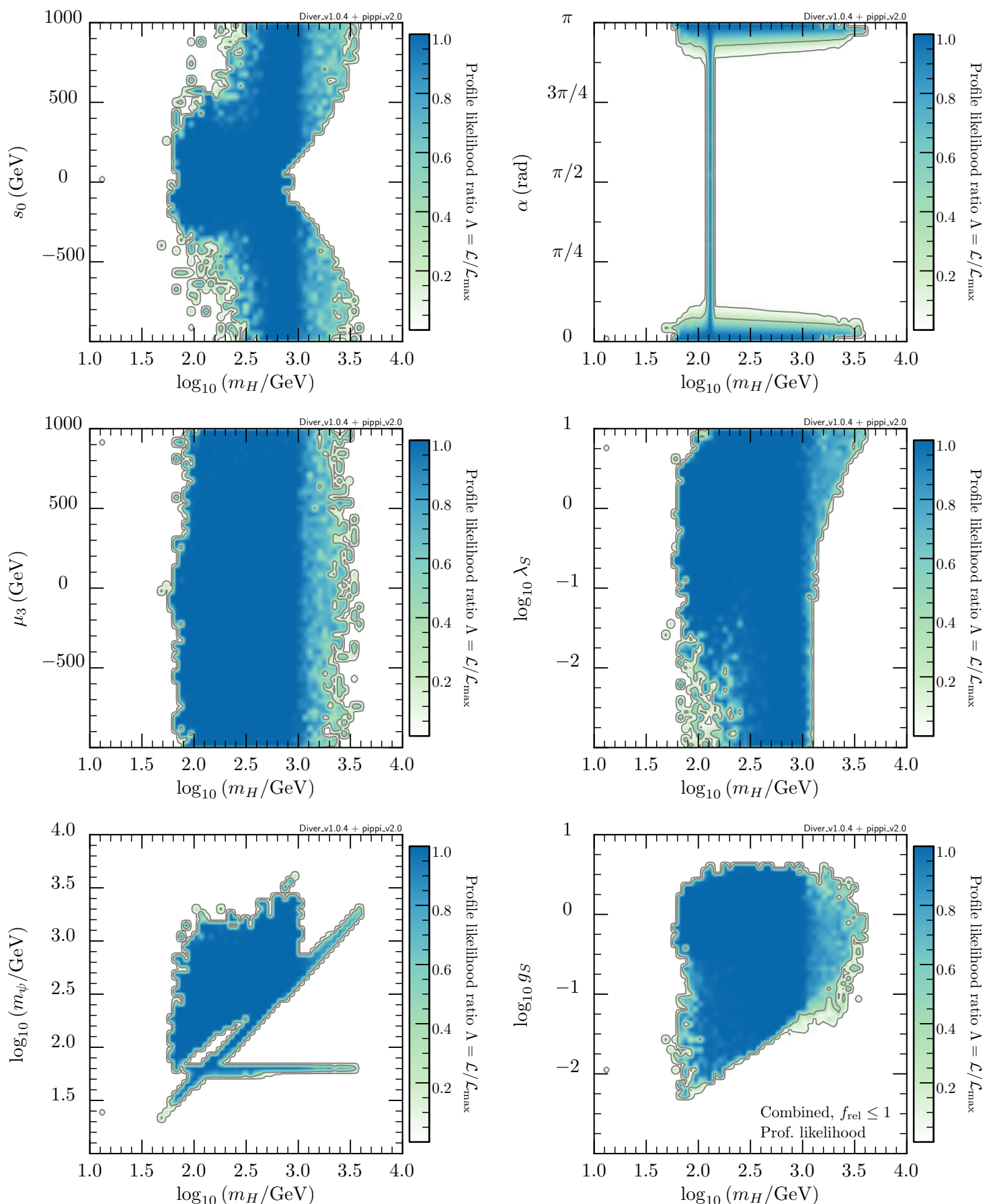

Figure 4. 2D PL plots from a global fit of our model assuming $f_{\text {rel }} \equiv \Omega_{\psi} / \Omega_{\mathrm{DM}} \leq 1$. The contour lines mark out the $1 \sigma(68.3 \%)$ and $2 \sigma(95.4 \%)$ C.L. regions.

2. $\left(m_{H}, \alpha\right)$ plane: we see that the model is allowed by all constraints for a range of low and high $m_{H}$ values provided $\alpha \simeq 0, \pi$. This is expected as the second scalar $H$ is decoupled in this regime and gives no new contribution to the observed Higgs signal strengths. However, when $m_{H} \simeq m_{h}$, the two scalars are indistinguishable from the 
point of direct Higgs searches and Higgs signal strength measurements. As is evident in eqs. (3.14) and (3.27), direct detection and EWPO constraints respectively are also relaxed in this regime. The net result is that all values of $\alpha$ are allowed when $m_{H} \simeq m_{h}$.

3. $\left(m_{H}, \mu_{3}\right)$ and $\left(m_{H}, \lambda_{S}\right)$ planes: these parameter planes are mostly unconstrained by our global fit except for $m_{H} \lesssim m_{h} / 2$ (excluded by the Higgs signal strength measurements) and $m_{H} \gtrsim 4 \mathrm{TeV}$ (ruled out by the EWBG constraint). For $m_{H} \gtrsim$ $1.3 \mathrm{TeV}$, large values of $\lambda_{S}$ are required to facilitate a successful EWBG.

4. $\left(m_{H}, m_{\psi}\right)$ plane: for $m_{\psi} \lesssim m_{h} / 2$, the fermion DM can only annihilate into light SM quarks, thereby giving $f_{\text {rel }}>1$. On the other hand, $m_{\psi} \gtrsim m_{h} / 2$ is constrained by the DM relic density and XENON1T limits. When $m_{\psi} \simeq m_{h} / 2$, all values of $m_{H}$ up to $\sim 4 \mathrm{TeV}$ are allowed by the Planck measured relic density and XENON1T limits; this region appears in the plot as a horizontal band. In this band, small values of $g_{S}$ can yield a fermion DM relic density and DM-nucleon cross-section that is compatible with the Planck measured value and XENON1T limit respectively.

For $m_{\psi} \in\left[m_{h} / 2, m_{H} / 2\right]$, the region is disfavoured by either the Planck measured relic density or XENON1T limit. This is generally expected from an incompatibility between small values of $g_{S}$ which are favoured by the XENON1T limit (as it gives a small DM-nucleon cross-section $\sigma_{\mathrm{SI}}^{\psi \mathcal{N}}$ ) but disfavoured by the relic density constraint (as it gives $f_{\text {rel }}>1$ ) and vice versa.

The diagonal band corresponds to the second resonance $m_{\psi} \simeq m_{H} / 2$. Similar to the first resonance $m_{\psi} \simeq m_{h} / 2$, all points in this band are allowed by the relic density and XENON1T limits. As $g_{S}$ is profiled over, small values of $g_{S}$ can easily give $f_{\text {rel }} \leq 1$ and $\sigma_{\mathrm{SI}}^{\text {eff }} \leq \sigma_{\text {XENON1T }}$. On the other hand, when $m_{H} \gtrsim 4 \mathrm{TeV}$, parameter points are disfavoured by the EWPO and EWBG constraints. For $m_{\psi} \gtrsim 3.2 \mathrm{TeV}$, the region is robustly excluded by the combined constraints.

5. $\left(m_{H}, g_{S}\right)$ plane: in this plane, a lower limit on $g_{S}$ comes from the DM relic density constraint as smaller values of $g_{S}$ lead to an overabundance of the fermion DM in the universe today. This lower limit becomes weaker as $m_{H}$ increases. For $m_{H} \gtrsim 4 \mathrm{TeV}$, the coupling $\lambda_{\Phi S}$ becomes non-perturbative, thus this region is disfavoured. Similarly, values of $g_{S} \gtrsim 3.2$ are disfavoured by the EWBG constraint as they lead to runaway directions in the potential, see figure 3 .

In figure 5, we show the $1 \mathrm{D}$ PL plots for the parameters $m_{H}, m_{\psi}$ and $g_{S}{ }^{8}$ From these plots, it is evident that the combined constraints impose an upper and lower limit on $m_{H}$, $m_{\psi}$ and $g_{S}$, namely

$$
m_{h} / 2 \lesssim m_{H} \lesssim 5 \mathrm{TeV}, \quad 32 \mathrm{GeV} \lesssim m_{\psi} \lesssim 3.2 \mathrm{TeV}, \quad 5.6 \times 10^{-3} \lesssim g_{S} \lesssim 3.5 .
$$

\footnotetext{
${ }^{8}$ For the remaining parameters, we find that the PL ratio $\Lambda$ is roughly flat and equal to 1 at all values. In other words, the parameters $s_{0}, \mu_{3}, \lambda_{S}$ and $\alpha$ are unconstrained by our global fit.
} 

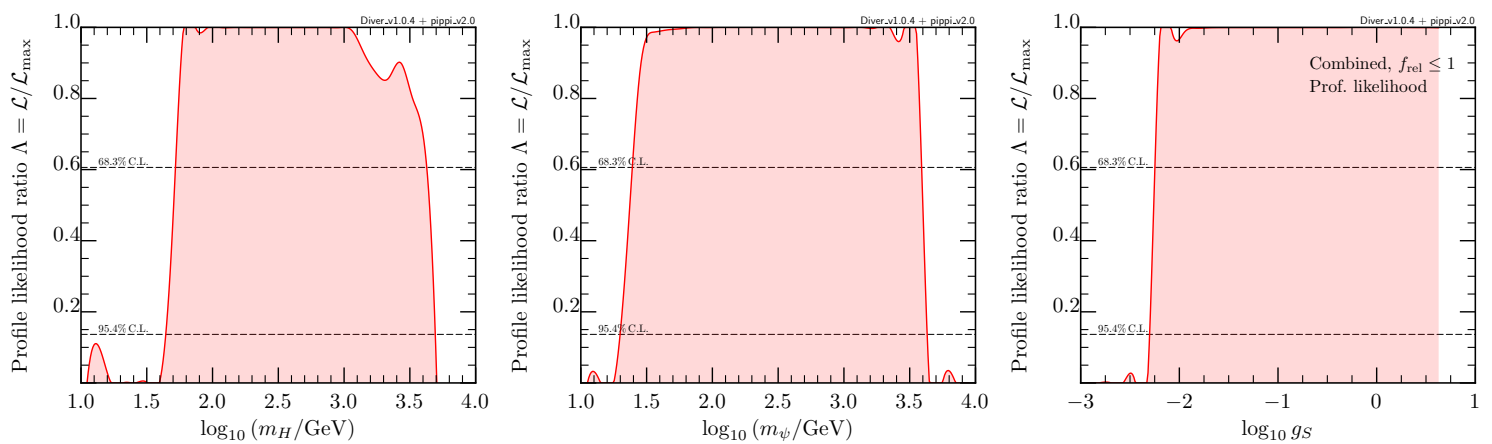

Figure 5. 1D PL plots for $m_{H}$ (left), $m_{\psi}$ (center) and $g_{S}$ (right) assuming $f_{\text {rel }} \leq 1$. The respective plots for $s_{0}, \mu_{3}$ and $\lambda_{S}$ are not shown as they are unconstrained by our global fit.
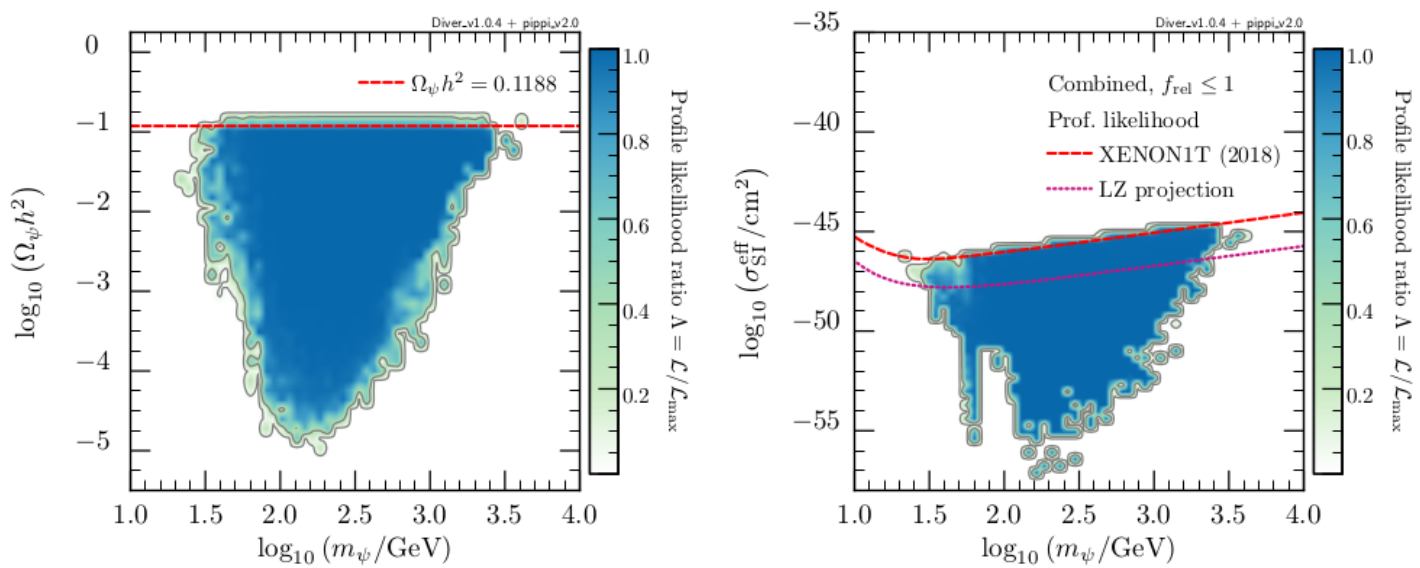

Figure 6. Left panel: fermion DM relic density vs. the fermion DM mass. The red dashed curve corresponds to the Planck measured value [67]. Right panel: effective SI DM-nucleon cross-section vs. the fermion DM mass. The red dashed curve shows the current $90 \%$ C.L. upper limit from the XENON1T (2018) [68], whereas the violet dotted curve shows the projected sensitivity of the LUX-ZEPLIN (LZ) [122] experiment.

These limits are based on our chosen ranges and priors for the free model parameters (as summarised in table 1). For instance, our lower limit on $m_{H}$ can be softened by reducing the branching ratio $\mathcal{B R}(h \rightarrow H H)$ when $\alpha=0, \pi$. In these cases, the reduced branching ratio can give a better fit to the observed signal strength measurements for a SM-like Higgs boson $h$. For non-zero mixing angles, however, this part of the parameter space is strongly constrained by the direct Higgs searches performed at the LEP experiment.

In figure 6, we show the key observables such as the fermion DM relic density (left panel) and effective SI DM-nucleon cross-section (right panel). These can be compared against the Planck measured value and XENON1T limit. It is evident that all of the sampled points satisfy $f_{\text {rel }} \leq 1$ and $\sigma_{\text {SI }}^{\text {eff }} \leq \sigma_{\text {XENON1T. }}$. We also show the projected sensitivity of the LUX-ZEPLIN (LZ) [122] experiment. Intriguingly, the LZ experiment will probe 2 orders of magnitude smaller DM-nucleon cross sections than the XENON1T experiment. Due to the two resonances $m_{\psi} \simeq m_{h, H} / 2$ and the ability to profile over $\alpha$, the direct 

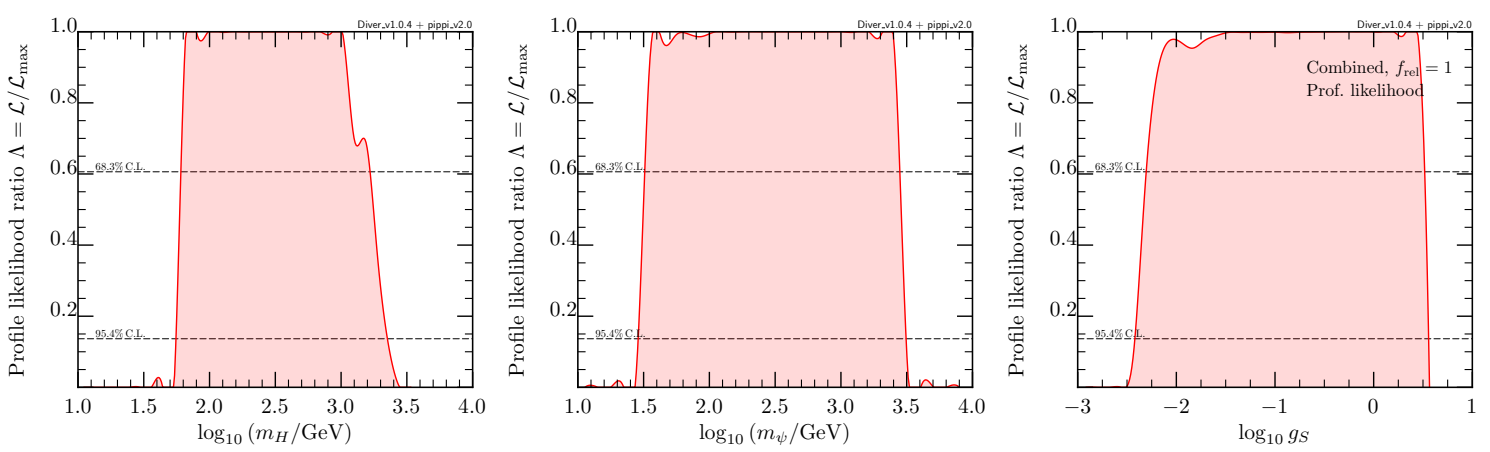

Figure 7. Same as figure 5 except for the $f_{\text {rel }}=1$ case.

detection cross-section in our model can be significantly suppressed to avoid bounds from current and future direct search experiments.

\subsubsection{Scenario II: $f_{\text {rel }}=1$}

In this subsection, we present results from our global fit assuming $f_{\text {rel }}=1$. The only difference with respect to the $f_{\text {rel }} \leq 1$ case is our use of a Gaussian likelihood function for the Planck measured DM relic density. In this case, not only small values of $g_{S}$ are disfavoured by the relic density constraint (as they give $f_{\text {rel }}>1$ ), large values of $g_{S}$ are also disfavoured (as they give $f_{\text {rel }} \ll 1$ ).

The resulting 1D and 2D PL plots from our 7D scans are shown in figures 7 and 8 respectively. In comparison with figures 5 and 4 respectively, the shape of the 1 and $2 \sigma$ C.L. contours is mostly similar; thus we refer to subsection 4.2.1 to avoid repetition. However, the allowed parameter space is significantly smaller. This is expected as the allowed region not only has to reproduce the observed DM abundance, it also has to yield a successful EWBG.

In general, we find that our model can easily satisfy all constraints provided $\alpha \simeq 0$, $\pi$. This is expected as the new scalar $H$ is decoupled in this regime and provides no new contribution to the observed Higgs signal strength measurements. Thus, the allowed final states from the fermion DM annihilation are $h h, H H$ and $h H$, which gives $f_{\text {rel }}=1$.

An important point to note is that in the $\mathbb{Z}_{2}$ symmetric case [36], the scalar Higgs portal coupling cannot simultaneously explain the observed DM abundance and matterantimatter asymmetry. This is expected as large values of the portal coupling are required to yield a successful EWBG, whereas small values are required to satisfy the direct detection limits. In contrast, our model contains additional couplings (e.g., $\mu_{3}$ and $\mu_{\Phi S}$ ) between the new scalar $S$ and SM Higgs boson; these couplings can aid in generating a strong first-order EWPT. As $\mu_{3}$ and $\mu_{\Phi S}$ does not significantly affect the phenomenology of the fermion DM (which is mostly determined by $g_{S}$ and $\alpha$ ), the fermion DM can easily saturate the observed DM abundance. These two features together allow the model to simultaneously explain the observed DM abundance and matter-antimatter asymmetry. 

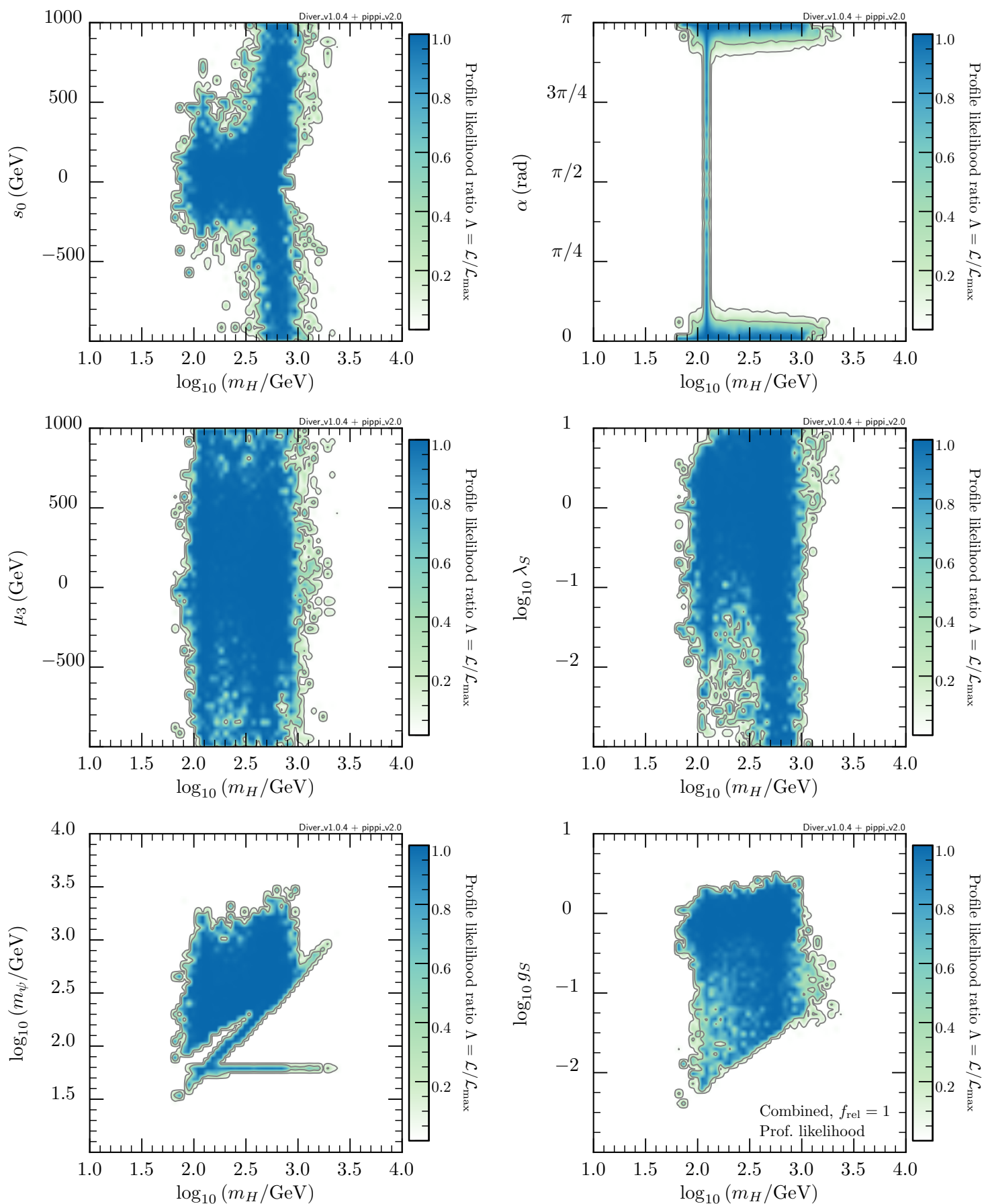

Figure 8. Same as figure 4 except for the $f_{\text {rel }}=1$ case.

\subsection{Gravitational wave signals}

The computation of gravitational wave (GW) signals requires a detailed study of the dynamics of the phase transition (PT). Luckily, the analysis of bubble nucleation is to some extent generic, and the steps required are always similar, albeit using a different scalar 
potential. In our model, the main difficulty is that the transition always involves both scalar fields, and finding a correct tunnelling path in the 2D field space is always necessary. We tackle this problem in the same way as in ref. [36]. In particular, we use the method described there to find the appropriate tunnelling path and the bubble solutions which drive the transition in each case. The main drawback of this calculation is that it is computationally expensive when compared to all other constraints discussed in section 3 . Thus, we first identify interesting points in the model parameter space from our global fit, and check the detailed PT dynamics and GW signals afterwards.

For each viable point from our global fit assuming $f_{\text {rel }} \leq 1$, we compute the tunnelling path and corresponding action as in ref. [36]. Next, we compute the fraction of volume converted to the true vacuum [102] to accurately calculate the bubble percolation temperature $T_{p}$. This allows us to identify cases in which too strong supercooling renders percolation impossible; as temperature drops, the false vacuum energy dominates the expansion of the universe and an inflationary phase begins $[102,123,124]$. We find that a significant number of interesting points are excluded as the decay is too suppressed and the transition never successfully finishes. This happens because the extended parameter space with respect to the simple scalar potential in ref. [36] allows for a formation of a large tree-level barrier which can persist even at $T=0$ and suppress the vacuum decay probability. In our 7D scans, we only use the approximation involving the critical temperature $T_{c}$ at which the symmetric and EWSB minima are degenerate. Such points are perfectly valid and predict $v_{c} / T_{c}>1$ as required for a successful EWBG. However, after a more detailed analysis, we find that roughly $50 \%$ of all points remain viable and their GW spectra have large enough amplitudes to be shown in our plots.

For the viable parameter points, we calculate the ratio of the released latent heat to the energy density of the plasma background, $\alpha_{\mathrm{GW}},{ }^{9}$ and the size of bubbles carrying the most energy at percolation $R_{\mathrm{MAX}}$, which we then convert to the more familiar inverse time of the phase transition $\beta / H=(8 \pi)^{1 / 3} v_{w} /\left(H R_{\mathrm{MAX}}\right)[102,125]$. These two parameters are essential for computing the GW spectra $[11,97]$. We also assume that the speed of bubble walls is close to the speed of light $\left(v_{w} / c \approx 1\right)$ which is valid for the very strong first-order PTs that we are mostly interested in.

Our calculation of the GW spectrum is based on ref. [102]. In particular, we do not include the signal contribution from collisions of bubbles [126-129] as the bubbles reach equilibrium with the surrounding plasma and most of the energy is pumped into fluid shells around them [130]. The two remaining sources are sound waves in the plasma [131-134] and turbulence [135-138] ensuing after the sound waves period. We also check the condition for the sound waves to last more than one Hubble time which was assumed to hold while obtaining the GW spectra in references above. We show this criterion in the $\left(\alpha_{\mathrm{GW}}, \beta / H\right)$ plane (see refs. [102, 134] for more details) along with our results in figure 9, assuming $v_{w} / c \approx 1$ which results in the largest allowed parameter space. We find that no parameter points are consistent with this criterion. This implies that the standard formula for sound wave spectra [11] is probably overestimating the true signal. On the other hand, the

\footnotetext{
${ }^{9}$ Not to be confused with the mixing angle $\alpha$.
} 


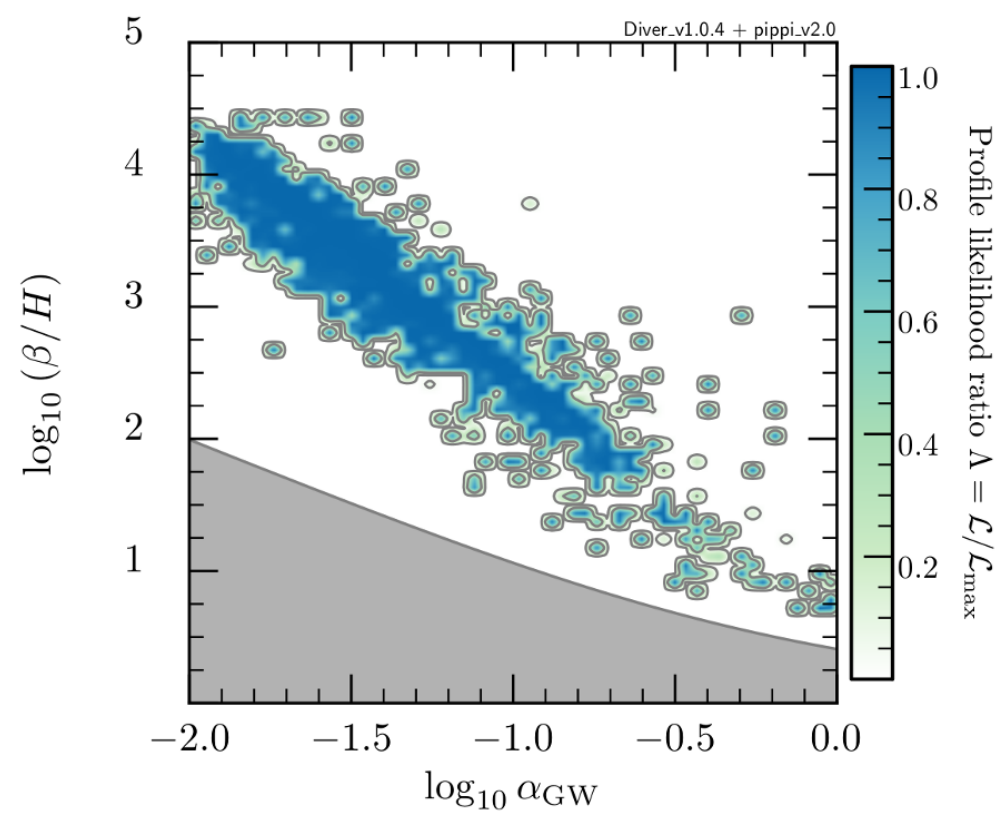

Figure 9. Viable points from our global fit assuming $f_{\text {rel }} \leq 1$ in terms of the parameters $\alpha_{\mathrm{GW}}$ and $\beta / H$. The grey region shows where the sound waves last more than a Hubble time (assuming $v_{w} / c \approx 1$ which results in the largest allowed area) and reliably produce a GW signal.

turbulence signal will be stronger than the standard estimate as the turbulent motion begins more promptly after the PT.

In figure 10, we show the resulting GW spectra of viable points as sourced by sound waves (top panel) and turbulence (bottom panel), and their dependence on the percolation temperature $T_{p}$. As the condition to reliably generate a GW signal from sound waves is not fulfilled, a dedicated numerical simulation would be necessary to ascertain the spectral shape. We expect that the final results will lie somewhere between these two figures. In these figures, we discarded points with almost identical GW parameters to avoid plotting many overlapping results. Thus, we only show 100 representative lines out of 10,000 GW spectra as computed from our results. We also show the detection prospects of Laser Interferometer Space Antenna (LISA) [139] (assuming the most optimistic A5M5 configuration), Deci-hertz Interferometer Gravitational-wave Observatory (DECIGO) and Big Bang Observer (BBO) [140]. The current and future sensitivity bands of LIGO [141-143], the European Pulsar Timing Array (EPTA) [144], the Square Kilometre Array (SKA) [145], Cosmic Explorer (CE) [146] and the Einstein Telescope (ET) $[147,148]$ fall in a different frequency range than that of the viable points. Thus, these experiments give no hope for detection of any of our results.

In summary, we find that the GW spectra of viable points that are interesting from the point of view of baryogenesis can lie within reach of future GW experiments such as LISA, DECIGO and BBO. However, the uncertainty of the sound wave spectrum can have a dramatic impact on the results. In the overly optimistic case of the standard sound wave signal, roughly $15 \%$ of all of our viable points would be detectable by LISA while for the 


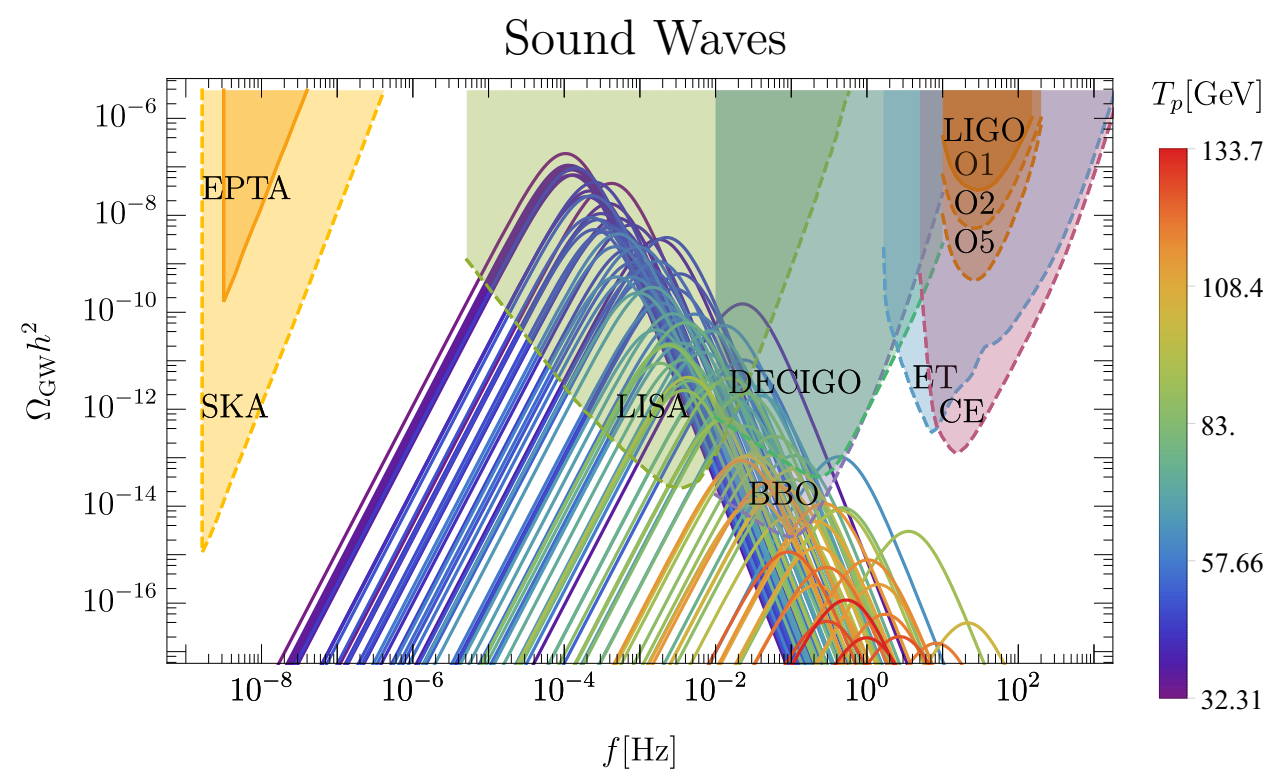

\section{Turbulence}

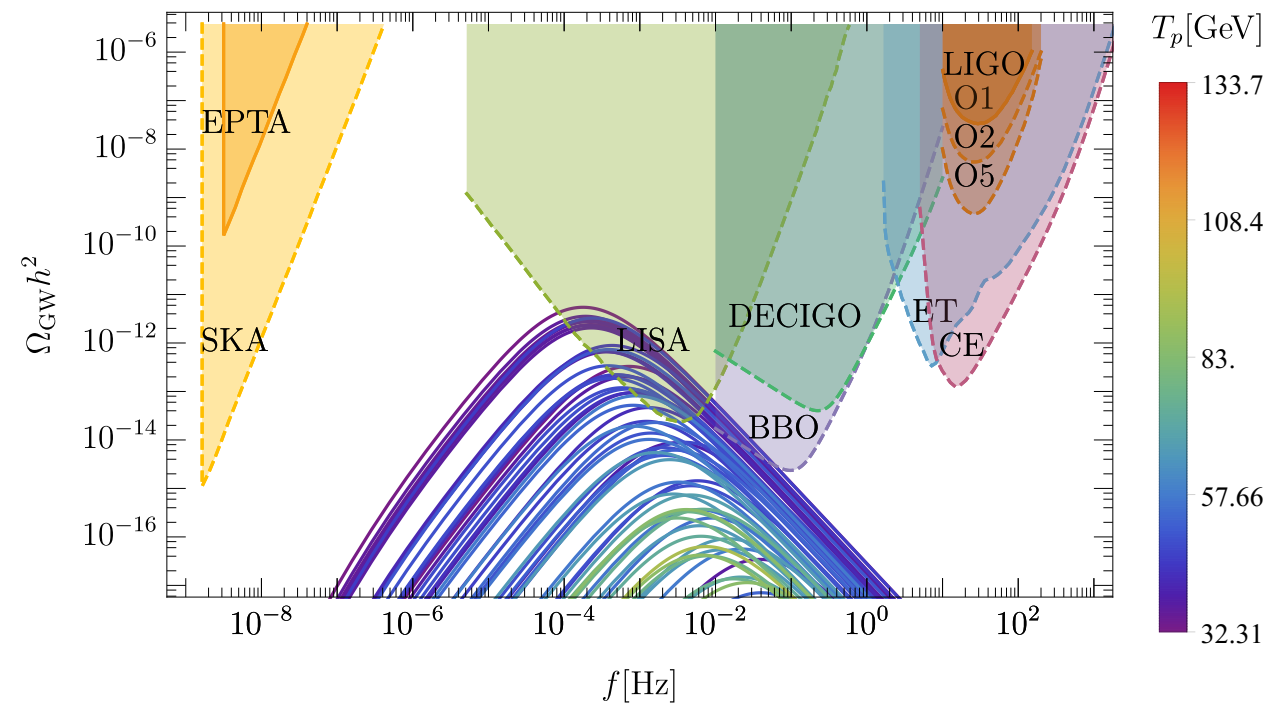

Figure 10. Gravitational wave $(\mathrm{GW})$ spectra of viable points as sourced by sound waves (top panel) and turbulence (bottom panel) along with their dependence on the percolation temperature $T_{p}$. Current sensitivity bands of LIGO and EPTA, as well as detection prospects of LISA, DECIGO, $\mathrm{BBO}$ and SKA are also shown for comparison (see text for more details).

most pessimistic turbulence-only spectrum, this number falls below half a percent. We also confirm that pulsar timing arrays and terrestrial experiments (e.g., LIGO) are not sensitive to frequencies that result in a GW signal from a strong EWPT. Notably, our results are qualitatively very similar to the $\mathbb{Z}_{2}$ symmetric ones in ref. [102], despite our general non- $\mathbb{Z}_{2}$ symmetric potential. This leads us to believe that our current knowledge of the Higgs boson properties (most notably, a constraint on the mixing angle $\alpha$ ) is enough to significantly constrain viable potentials, and bring them closer to the $\mathbb{Z}_{2}$ symmetric case. 


\section{Conclusions}

In this paper, we have performed the most comprehensive and up-to-date study of the extended scalar singlet model with a fermionic DM candidate. After performing a 7D scan of the model using only the EWBG constraint, we found regions in the model parameter space that can facilitate a successful EWBG. From our 1D PL plots, we showed that a successful EWBG can be viable in all parts of the model parameter space provided $g_{S} \lesssim 5.62$.

After building intuition from the EWBG only results, we performed a global fit of our model using the constraints from the Planck measured DM relic density, direct detection limits from the XENON1T (2018) experiment, electroweak precision observables (EWPO) and Higgs searches at colliders. This allowed us to constrain parts of the 7D model parameter space. In particular, our global fit placed an upper and lower limit on $m_{H}, m_{\psi}$ and $g_{S}$, namely $m_{h} / 2 \lesssim m_{H} \lesssim 5 \mathrm{TeV}, 32 \mathrm{GeV} \lesssim m_{\psi} \lesssim 3.2 \mathrm{TeV}$ and $5.6 \times 10^{-3} \lesssim g_{S} \lesssim 3.5$. Moreover, we confirmed that our model can simultaneously yield a strong first-order phase transition and saturate the observed DM abundance. This is an important feature which is missing in the $\mathbb{Z}_{2}$ symmetric case.

From the viable points that satisfied all of the available constraints, we computed the GW spectra, and checked the discovery prospects of the model at current and future GW experiments. In doing so, we found that the GW spectra of viable points can be within reach of future GW experiments such as LISA, DECIGO and BBO. We checked that the condition for sound waves to be a long-lasting source of GWs is not satisfied for any of our results. This implies that the standard sound wave spectrum used in the literature likely overestimates the true signal, whereas the turbulence signal can be stronger than the standard prediction as the turbulence sets in quicker after the end of the phase transition. Unfortunately, the overall result will still likely be a reduction of the overall spectrum, thereby reducing the discovery prospects. Specifically, in our results we find that $15 \%$ of our viable points would be within the reach of LISA if the final spectrum was close to the standard sound wave prediction. However, this number falls down to less than half a percent in the most pessimistic case of only a turbulence-sourced GW signal.

\section{Acknowledgments}

We thank Philip Bechtle, Malcolm Fairbairn, José M. No, Chris Rogan and Pat Scott for helpful discussions. AB thanks Sean Crosby from the CoEPP Research Computing for providing computing assistance and allocating resources. This work was supported by the Swedish Research Council (contract 621-2014-5772), ARC Centre of Excellence for Particle Physics at the Terascale (CoEPP) (CE110001104) and the Centre for the Subatomic Structure of Matter (CSSM). This work also made use of the supercomputing resources provided by the Phoenix HPC service at the University of Adelaide. ML was supported in part by the Polish MNiSW grant IP2015 043174 and STFC grant number ST/L000326/1. AB was supported by the Australian Postgraduate Award (APA). MW is 
supported by the Australian Research Council Future Fellowship FT140100244. Feynman diagrams were drawn using the TikZ-Feynman_v1.1.0 package [149].

\section{A Tree-level scalar potential}

The tree-level scalar potential in eq. (2.5) expands to

$$
V_{\text {tree }}=-\mu_{\Phi}^{2} \Phi^{\dagger} \Phi+\lambda_{\Phi}\left(\Phi^{\dagger} \Phi\right)^{2}-\frac{1}{2} \mu_{S}^{2} S^{2}-\frac{1}{3} \mu_{3} S^{3}+\frac{1}{4} \lambda_{S} S^{4}+\mu_{\Phi S} \Phi^{\dagger} \Phi S+\frac{1}{2} \lambda_{\Phi S} \Phi^{\dagger} \Phi S^{2} .
$$

With the following definitions

$$
\Phi=\left(\begin{array}{c}
G^{+} \\
\frac{1}{\sqrt{2}}\left(\phi+i G^{0}\right)
\end{array}\right), \quad \Phi^{\dagger}=\left(G^{-}, \frac{1}{\sqrt{2}}\left(\phi-i G^{0}\right)\right),
$$

where $G^{-} \equiv\left(G^{+}\right)^{*}$, the potential in eq. (A.1) depends on 2 complex $\left(G^{+}, G^{-}\right)$and 3 real $\left(G^{0}, \phi, S\right)$ scalar fields.

After EWSB, the $\phi$ and $S$ fields acquire their VEVs in eq. (2.8). Thus, the following partial derivatives

$$
\frac{\partial V_{\text {tree }}}{\partial G^{0}}, \frac{\partial V_{\text {tree }}}{\partial G^{-}}, \frac{\partial V_{\text {tree }}}{\partial G^{+}}, \frac{\partial V_{\text {tree }}}{\partial \phi}, \frac{\partial V_{\text {tree }}}{\partial S},
$$

must vanish at the EWSB minimum $\left(\left.\langle\phi\rangle\right|_{T=0},\left.\langle S\rangle\right|_{T=0}\right)=\left(v_{0}, s_{0}\right)$. This gives

$$
\begin{aligned}
& 0=\left.\frac{\partial V_{\text {tree }}}{\partial G^{0}}\right|_{\left(v_{0}, s_{0}\right)}=\left.\frac{\partial V_{\text {tree }}}{\partial G^{-}}\right|_{\left(v_{0}, s_{0}\right)}=\left.\frac{\partial V_{\text {tree }}}{\partial G^{+}}\right|_{\left(v_{0}, s_{0}\right)}, \\
& 0=\left.\frac{\partial V_{\text {tree }}}{\partial \phi}\right|_{\left(v_{0}, s_{0}\right)}=-\mu_{\Phi}^{2} v_{0}+\lambda_{\Phi} v_{0}^{3}+\mu_{\Phi S} s_{0} v_{0}+\frac{1}{2} \lambda_{\Phi S} v_{0} s_{0}^{2}, \\
& 0=\left.\frac{\partial V_{\text {tree }}}{\partial S}\right|_{\left(v_{0}, s_{0}\right)}=-\mu_{S}^{2} s_{0}-\mu_{3} s_{0}^{2}+\lambda_{S} s_{0}^{3}+\frac{1}{2} \mu_{\Phi S} v_{0}^{2}+\frac{1}{2} \lambda_{\Phi S} s_{0} v_{0}^{2} .
\end{aligned}
$$

A simple rearrangement gives us the following EWSB conditions

$$
\begin{aligned}
& \mu_{\Phi}^{2}=\lambda_{\Phi} v_{0}^{2}+\mu_{\Phi S} s_{0}+\frac{1}{2} \lambda_{\Phi S} s_{0}^{2}, \\
& \mu_{S}^{2}=-\mu_{3} s_{0}+\lambda_{S} s_{0}^{2}+\frac{\mu_{\Phi S} v_{0}^{2}}{2 s_{0}}+\frac{1}{2} \lambda_{\Phi S} v_{0}^{2} .
\end{aligned}
$$

Now, we compute the second-order partial derivatives at the EWSB minimum. The only non-zero ones are given by

$$
\begin{aligned}
\left.\frac{\partial^{2} V_{\text {tree }}}{\partial G^{0} \partial G^{0}}\right|_{\left(v_{0}, s_{0}\right)}=\left.\frac{\partial^{2} V_{\text {tree }}}{\partial G^{-} \partial G^{+}}\right|_{\left(v_{0}, s_{0}\right)} & =\left.\frac{\partial^{2} V_{\text {tree }}}{\partial G^{+} \partial G^{-}}\right|_{\left(v_{0}, s_{0}\right)}=-\mu_{\Phi}^{2}+\lambda_{\Phi} v_{0}^{2}+\mu_{\Phi S} s_{0}+\frac{1}{2} \lambda_{\Phi S} s_{0}^{2}, \\
\left.\frac{\partial^{2} V_{\text {tree }}}{\partial \phi^{2}}\right|_{\left(v_{0}, s_{0}\right)} & =-\mu_{\Phi}^{2}+3 \lambda_{\Phi} v_{0}^{2}+\mu_{\Phi S} s_{0}+\frac{1}{2} \lambda_{\Phi S} s_{0}^{2}, \\
\left.\frac{\partial^{2} V_{\text {tree }}}{\partial S^{2}}\right|_{\left(v_{0}, s_{0}\right)} & =-\mu_{S}^{2}-2 \mu_{3} s_{0}+3 \lambda_{S} s_{0}^{2}+\frac{1}{2} \lambda_{\Phi S} v_{0}^{2}, \\
\left.\frac{\partial^{2} V_{\text {tree }}}{\partial \phi \partial S}\right|_{\left(v_{0}, s_{0}\right)}=\left.\frac{\partial^{2} V_{\text {tree }}}{\partial S \partial \phi}\right|_{\left(v_{0}, s_{0}\right)} & =\mu_{\Phi S} v_{0}+\lambda_{\Phi S} v_{0} s_{0} .
\end{aligned}
$$


Using eqs. (A.2) and (A.3), these expressions can be simplified to

$$
\begin{aligned}
\left.\frac{\partial^{2} V_{\text {tree }}}{\partial G^{0} \partial G^{0}}\right|_{\left(v_{0}, s_{0}\right)}=\left.\frac{\partial^{2} V_{\text {tree }}}{\partial G^{-} \partial G^{+}}\right|_{\left(v_{0}, s_{0}\right)} & =\left.\frac{\partial^{2} V_{\text {tree }}}{\partial G^{+} \partial G^{-}}\right|_{\left(v_{0}, s_{0}\right)}=0 \\
\left.\frac{\partial^{2} V_{\text {tree }}}{\partial \phi^{2}}\right|_{\left(v_{0}, s_{0}\right)} & =2 \lambda_{\Phi} v_{0}^{2} \\
\left.\frac{\partial^{2} V_{\text {tree }}}{\partial S^{2}}\right|_{\left(v_{0}, s_{0}\right)} & =-\mu_{3} s_{0}+2 \lambda_{S} s_{0}^{2}-\frac{\mu_{\Phi S} v_{0}^{2}}{2 s_{0}} \\
\left.\frac{\partial^{2} V_{\text {tree }}}{\partial \phi \partial S}\right|_{\left(v_{0}, s_{0}\right)}= & \left.\frac{\partial^{2} V_{\text {tree }}}{\partial S \partial \phi}\right|_{\left(v_{0}, s_{0}\right)}=\mu_{\Phi S} v_{0}+\lambda_{\Phi S} v_{0} s_{0}
\end{aligned}
$$

After EWSB, the $\phi$ and $S$ fields can be expanded as

$$
\phi=v_{0}+\varphi, \quad S=s_{0}+s .
$$

As $\partial V_{\text {tree }} / \partial \phi=\partial V_{\text {tree }} / \partial \varphi$ and $\partial V_{\text {tree }} / \partial S=\partial V_{\text {tree }} / \partial s$, a mass-term for the real scalar fields $\mathcal{A}^{T}=(\varphi, s)$ is

$$
\mathscr{L}_{\text {mass-term }}=-\frac{1}{2} \mathcal{A}^{T} \mathcal{M}^{2} \mathcal{A}
$$

where

$$
\mathcal{M}^{2}=\left(\begin{array}{ll}
\mathcal{M}_{\varphi \varphi}^{2} & \mathcal{M}_{\varphi s}^{2} \\
\mathcal{M}_{s \varphi}^{2} & \mathcal{M}_{s s}^{2}
\end{array}\right) \equiv\left(\begin{array}{ll}
\left.\frac{\partial^{2} V_{\text {tree }}}{\partial \varphi^{2}}\right|_{\left(v_{0}, s_{0}\right)} & \left.\frac{\partial^{2} V_{\text {tree }}}{\partial \varphi \partial s}\right|_{\left(v_{0}, s_{0}\right)} \\
\left.\frac{\partial^{2} V_{\text {tree }}}{\partial s \partial \varphi}\right|_{\left(v_{0}, s_{0}\right)} & \left.\frac{\partial^{2} V_{\text {tree }}}{\partial s^{2}}\right|_{\left(v_{0}, s_{0}\right)}
\end{array}\right)
$$

is the squared mass matrix. Using eqs. (A.5)-(A.7), the matrix elements are given by

$$
\mathcal{M}_{\varphi \varphi}^{2}=2 \lambda_{\Phi} v_{0}^{2}, \quad \mathcal{M}_{s s}^{2}=-\mu_{3} s_{0}+2 \lambda_{S} s_{0}^{2}-\frac{\mu \Phi S v_{0}^{2}}{2 s_{0}}, \quad \mathcal{M}_{\varphi s}^{2}=\mathcal{M}_{s \varphi}^{2}=\mu_{\Phi S} v_{0}+\lambda_{\Phi S} v_{0} s_{0}
$$

For the EWSB minimum to be a stable (i.e., not a saddle point) solution of eq. (A.1), the symmetric $5 \times 5$ Hessian matrix $\mathcal{H}$ must be positive-definite. At the EWSB minimum, it is given by

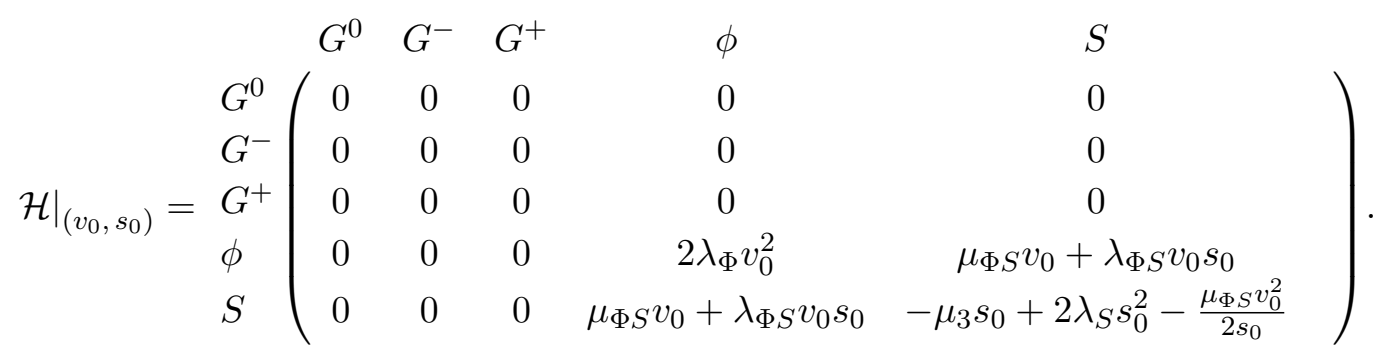

The above matrix is guaranteed to be positive-definite if the determinant (eigenvalue) of the $2 \times 2$ sub-matrix is non-zero (positive). These two requirements give

$$
\lambda_{\Phi}>0, \quad 2 \lambda_{S} s_{0}^{2}-\left(\mu_{3} s_{0}+\frac{\mu_{\Phi S} v_{0}^{2}}{2 s_{0}}\right)>0 .
$$


To study the bounds of the tree-level scalar potential, eq. (A.1) can be expressed in terms of the $\phi$ and $S$ fields as

$$
V_{\text {tree }} \simeq-\frac{1}{2} \mu_{\Phi}^{2} \phi^{2}+\frac{1}{4} \lambda_{\Phi} \phi^{4}-\frac{1}{2} \mu_{S}^{2} S^{2}-\frac{1}{3} \mu_{3} S^{3}+\frac{1}{4} \lambda_{S} S^{4}+\frac{1}{2} \mu_{\Phi S} \phi^{2} S+\frac{1}{4} \lambda_{\Phi S} \phi^{2} S^{2} .
$$

Depending on the chosen direction in the $(\phi, S)$ plane, three scenarios are possible.

1. Pure $\phi$ direction: in this case, the potential depends only on the $\phi$ field. It is bounded from below provided $\lambda_{\Phi}>0$.

2. Pure $S$ direction: in this case, the potential depends only on the $S$ field. It is bounded from below provided $\lambda_{S}>0$.

3. General $\phi$ and $S$ directions: at large $\phi$ and $S$ field values, the quartic terms in eq. (A.13) dominate. In this case, the potential can be approximated by

$$
\begin{aligned}
V_{\text {tree }} & \approx \frac{1}{4} \lambda_{\Phi} \phi^{4}+\frac{1}{4} \lambda_{S} S^{4}+\frac{1}{4} \lambda_{\Phi S} \phi^{2} S^{2} \\
& =\frac{1}{4} \lambda_{\Phi} \phi^{4}+\frac{1}{4} \lambda_{S}\left(S^{4}+\frac{\lambda_{\Phi S}}{\lambda_{S}} \phi^{2} S^{2}\right) \\
& =\frac{1}{4} \lambda_{\Phi} \phi^{4}+\frac{1}{4} \lambda_{S}\left(S^{4}+\frac{\lambda_{\Phi S}}{\lambda_{S}} \phi^{2} S^{2}+\frac{1}{4} \frac{\lambda_{\Phi S}^{2}}{\lambda_{S}^{2}} \phi^{4}\right)-\frac{1}{16} \frac{\lambda_{\Phi S}^{2}}{\lambda_{S}} \phi^{4} \\
& =\frac{1}{4}\left(\lambda_{\Phi}-\frac{1}{4} \frac{\lambda_{\Phi S}^{2}}{\lambda_{S}}\right) \phi^{4}+\frac{1}{4} \lambda_{S}\left(S^{2}+\frac{1}{2} \frac{\lambda_{\Phi S}}{\lambda_{S}} \phi^{2}\right)^{2} .
\end{aligned}
$$

Thus, the potential is bounded from below provided $\lambda_{S}>0$ and $\lambda_{\Phi S}>-2 \sqrt{\lambda_{\Phi} \lambda_{S}}$.

\section{B Mass eigenstate basis}

The squared mass matrix $\mathcal{M}^{2}$ is real and symmetric. It can be diagonalised by an orthogonal matrix $\mathcal{O}$. Thus, we define the mass eigenstates $(h, H)$ as

$$
\left(\begin{array}{l}
h \\
H
\end{array}\right)=\left(\begin{array}{cc}
\cos \alpha & -\sin \alpha \\
\sin \alpha & \cos \alpha
\end{array}\right)\left(\begin{array}{l}
\varphi \\
s
\end{array}\right) \text {. }
$$

The interaction eigenstates $(\varphi, s)$ are given by

$$
\left(\begin{array}{l}
\varphi \\
s
\end{array}\right)=\mathcal{O}\left(\begin{array}{c}
h \\
H
\end{array}\right), \quad \mathcal{O}=\left(\begin{array}{cc}
\cos \alpha & \sin \alpha \\
-\sin \alpha & \cos \alpha
\end{array}\right)
$$

Now, we consider the following matrix product

$$
\left(\begin{array}{ll}
\varphi & s
\end{array}\right) \mathcal{M}^{2}\left(\begin{array}{l}
\varphi \\
s
\end{array}\right)=\left(\begin{array}{ll}
h H & H
\end{array}\right) \mathcal{O}^{T} \mathcal{M}^{2} \mathcal{O}\left(\begin{array}{l}
h \\
H
\end{array}\right) \equiv\left(\begin{array}{ll}
h H
\end{array}\right) \mathcal{D}\left(\begin{array}{l}
h \\
H
\end{array}\right),
$$

where $\mathcal{D}=\operatorname{diag}\left(m_{h}^{2}, m_{H}^{2}\right)$ is a diagonal squared mass matrix for the physical mass eigenstates. Thus, the last equality in eq. (B.2) requires

$$
\mathcal{O}^{T} \mathcal{M}^{2} \mathcal{O}=\mathcal{D}
$$


The left-hand side of the above expression expands to

$$
\begin{aligned}
\mathcal{O}^{T} \mathcal{M}^{2} \mathcal{O} & =\left(\begin{array}{cc}
\cos \alpha & -\sin \alpha \\
\sin \alpha & \cos \alpha
\end{array}\right)\left(\begin{array}{ll}
\mathcal{M}_{\varphi \varphi}^{2} & \mathcal{M}_{\varphi s}^{2} \\
\mathcal{M}_{s \varphi}^{2} & \mathcal{M}_{s s}^{2}
\end{array}\right)\left(\begin{array}{cc}
\cos \alpha & \sin \alpha \\
-\sin \alpha & \cos \alpha
\end{array}\right) \\
& =\left(\begin{array}{cc}
\cos \alpha & -\sin \alpha \\
\sin \alpha & \cos \alpha
\end{array}\right)\left(\begin{array}{ll}
\mathcal{M}_{\varphi \varphi}^{2} \cos \alpha-\mathcal{M}_{\varphi s}^{2} \sin \alpha & \mathcal{M}_{\varphi \varphi}^{2} \sin \alpha+\mathcal{M}_{\varphi s}^{2} \cos \alpha \\
\mathcal{M}_{s \varphi}^{2} \cos \alpha-\mathcal{M}_{s s}^{2} \sin \alpha & \mathcal{M}_{s \varphi}^{2} \sin \alpha+\mathcal{M}_{s s}^{2} \cos \alpha
\end{array}\right) .
\end{aligned}
$$

As $\mathcal{M}_{\varphi s}^{2}=\mathcal{M}_{s \varphi}^{2}$, the elements of the $\mathcal{O}^{T} \mathcal{M}^{2} \mathcal{O}$ matrix are

$$
\begin{aligned}
& {\left[\mathcal{O}^{T} \mathcal{M}^{2} \mathcal{O}\right]_{11}=\mathcal{M}_{\varphi \varphi}^{2} \cos ^{2} \alpha+\mathcal{M}_{s s}^{2} \sin ^{2} \alpha-\mathcal{M}_{\varphi s}^{2} \sin 2 \alpha } \\
& {\left[\mathcal{O}^{T} \mathcal{M}^{2} \mathcal{O}\right]_{22}=\mathcal{M}_{\varphi \varphi}^{2} \sin ^{2} \alpha+\mathcal{M}_{s s}^{2} \cos ^{2} \alpha+\mathcal{M}_{\varphi s}^{2} \sin 2 \alpha } \\
{\left[\mathcal{O}^{T} \mathcal{M}^{2} \mathcal{O}\right]_{12}=} & {\left[\mathcal{O}^{T} \mathcal{M}^{2} \mathcal{O}\right]_{21}=-\frac{1}{2}\left(\mathcal{M}_{s s}^{2}-\mathcal{M}_{\varphi \varphi}^{2}\right) \sin 2 \alpha+\mathcal{M}_{\varphi s}^{2} \cos 2 \alpha }
\end{aligned}
$$

By equating these expressions to the elements of the diagonal matrix $\mathcal{D}$, we get

$$
\begin{aligned}
m_{h}^{2} & =\mathcal{M}_{\varphi \varphi}^{2} \cos ^{2} \alpha+\mathcal{M}_{s s}^{2} \sin ^{2} \alpha-\mathcal{M}_{\varphi s}^{2} \sin 2 \alpha, \\
m_{H}^{2} & =\mathcal{M}_{\varphi \varphi}^{2} \sin ^{2} \alpha+\mathcal{M}_{s s}^{2} \cos ^{2} \alpha+\mathcal{M}_{\varphi s}^{2} \sin 2 \alpha, \\
0 & =-\frac{1}{2}\left(\mathcal{M}_{s s}^{2}-\mathcal{M}_{\varphi \varphi}^{2}\right) \sin 2 \alpha+\mathcal{M}_{\varphi s}^{2} \cos 2 \alpha .
\end{aligned}
$$

The last equality can be conveniently expressed as

$$
\tan 2 \alpha=\frac{2 \mathcal{M}_{\varphi s}^{2}}{\mathcal{M}_{s s}^{2}-\mathcal{M}_{\varphi \varphi}^{2}} .
$$

We can rewrite eqs. (B.4)-(B.6) as the following matrix product

$$
\left(\begin{array}{c}
m_{h}^{2} \\
m_{H}^{2} \\
0
\end{array}\right)=\left(\begin{array}{ccc}
\cos ^{2} \alpha & \sin ^{2} \alpha & -2 \sin \alpha \cos \alpha \\
\sin ^{2} \alpha & \cos ^{2} \alpha & 2 \sin \alpha \cos \alpha \\
\sin \alpha \cos \alpha & -\sin \alpha \cos \alpha & \cos ^{2} \alpha-\sin ^{2} \alpha
\end{array}\right)\left(\begin{array}{l}
\mathcal{M}_{\varphi \varphi}^{2} \\
\mathcal{M}_{s s}^{2} \\
\mathcal{M}_{\varphi s}^{2}
\end{array}\right) .
$$

By computing the inverse of the above $3 \times 3$ matrix (i.e., by taking $\alpha \rightarrow-\alpha$ ), we get

$$
\left(\begin{array}{l}
\mathcal{M}_{\varphi \varphi}^{2} \\
\mathcal{M}_{s s}^{2} \\
\mathcal{M}_{\varphi s}^{2}
\end{array}\right)=\left(\begin{array}{ccc}
\cos ^{2} \alpha & \sin ^{2} \alpha & 2 \sin \alpha \cos \alpha \\
\sin ^{2} \alpha & \cos ^{2} \alpha & -2 \sin \alpha \cos \alpha \\
-\sin \alpha \cos \alpha & \sin \alpha \cos \alpha & \cos ^{2} \alpha-\sin ^{2} \alpha
\end{array}\right)\left(\begin{array}{c}
m_{h}^{2} \\
m_{H}^{2} \\
0
\end{array}\right) .
$$

From the above matrix product and eq. (A.11), we get

$$
\begin{aligned}
\lambda_{\Phi} & =\frac{\mathcal{M}_{\varphi \varphi}^{2}}{2 v_{0}^{2}}=\frac{1}{2 v_{0}^{2}}\left(m_{h}^{2} \cos ^{2} \alpha+m_{H}^{2} \sin ^{2} \alpha\right), \\
\mu_{\Phi S} & =-\frac{2 s_{0}}{v_{0}^{2}}\left(\mathcal{M}_{s s}^{2}+\mu_{3} s_{0}-2 \lambda_{S} s_{0}^{2}\right)=-\frac{2 s_{0}}{v_{0}^{2}}\left(m_{h}^{2} \sin ^{2} \alpha+m_{H}^{2} \cos ^{2} \alpha+\mu_{3} s_{0}-2 \lambda_{S} s_{0}^{2}\right), \\
\lambda_{\Phi S} & =\frac{1}{v_{0} s_{0}}\left(\mathcal{M}_{\varphi s}^{2}-\mu_{\Phi S} v_{0}\right)=\frac{1}{v_{0} s_{0}}\left[\left(m_{H}^{2}-m_{h}^{2}\right) \sin \alpha \cos \alpha-\mu_{\Phi S} v_{0}\right] .
\end{aligned}
$$




\section{Dark matter-nucleon coupling}

The interaction eigenstates $(\varphi, s)$ can be written in terms of the mass eigenstates $(h, H)$ as

$$
\left(\begin{array}{l}
\varphi \\
s
\end{array}\right)=\left(\begin{array}{cc}
\cos \alpha & \sin \alpha \\
-\sin \alpha & \cos \alpha
\end{array}\right)\left(\begin{array}{c}
h \\
H
\end{array}\right) \text {. }
$$

Thus, the scalar-fermion DM and quark Yukawa term in the SM Lagrangian expands to

$$
\begin{aligned}
\mathscr{L}_{\text {DM-quark }} & =-g_{S} \bar{\psi} \psi s-\sum_{q} \frac{m_{q}}{v_{0}} \varphi \bar{q} q \\
& =-g_{S} \bar{\psi} \psi(-\sin \alpha h+\cos \alpha H)-\sum_{q} \frac{m_{q}}{v_{0}}(\cos \alpha h+\sin \alpha H) \bar{q} q \\
& =g_{S} \sin \alpha \bar{\psi} \psi h-\frac{\cos \alpha}{v_{0}} \sum_{q} m_{q} h \bar{q} q-g_{S} \cos \alpha \bar{\psi} \psi H-\frac{\sin \alpha}{v_{0}} \sum_{q} m_{q} H \bar{q} q .
\end{aligned}
$$

In a typical direct detection experiment, the momentum transfer $q$ is roughly on the order of a few MeV. Assuming that the mediator masses $m_{h / H}$ are well above this value, ${ }^{10}$ i.e., $m_{h / H}^{2} \gg q^{2}$, we can safely approach direct detection in the context of an effective field theory (EFT) and integrate out the scalar mediators [150]. Thus, we can write down an effective DM-quark interaction Lagrangian as

$$
\mathscr{L}_{\mathrm{DM} \text {-quark }}^{\mathrm{eff}}=-\sum_{q} G_{q} \bar{\psi} \psi \bar{q} q
$$

where

$$
G_{q}=\frac{g_{S} \sin \alpha \cos \alpha}{v_{0}}\left(\frac{1}{m_{h}^{2}}-\frac{1}{m_{H}^{2}}\right) m_{q}
$$

is the effective DM-quark coupling.

In order to promote a DM-quark interaction to a DM-nucleon one, the quark contents of a nucleon must be taken into account. For a scalar mediator (as in our model), the quark Yukawa couplings generally scales with the mass of an interacting fermion. Thus, the dominant contribution comes from the strange quark content of a nucleon and from gluons via heavy quark loops. These contributions are parametrised by the hadronic matrix elements as

$$
f_{T q}^{(\mathcal{N})} \equiv \frac{m_{q}}{m_{\mathcal{N}}}\langle\mathcal{N}|\bar{q} q| \mathcal{N}\rangle,
$$

where $\mathcal{N} \in(p, n)$. For a pure scalar interaction, these matrix elements parametrise the contribution of a quark mass $m_{q}$ to the total mass of a nucleon $m_{\mathcal{N}}$. For more details on these parameters and recent estimates, see ref. [96] and references therein.

Using the heavy quark expansion [151], the contribution from gluons via heavy quark loops can be expressed in terms of the lighter quarks as

$$
f_{T c}^{(\mathcal{N})}=f_{T b}^{(\mathcal{N})}=f_{T t}^{(\mathcal{N})}=\frac{2}{27} f_{T G}^{(\mathcal{N})}=\frac{2}{27}\left(1-\sum_{q=u, d, s} f_{T q}^{(\mathcal{N})}\right) .
$$

\footnotetext{
${ }^{10}$ This is clearly the case for a SM-like Higgs boson with mass $m_{h}=125.13 \mathrm{GeV}$.
} 
Thus, we can write

$$
\frac{G_{\mathcal{N}}}{m_{\mathcal{N}}} \equiv \sum_{q} \frac{G_{q}}{m_{q}} f_{T q}^{(\mathcal{N})}=\sum_{q=u, d, s} \frac{G_{q}}{m_{q}} f_{T q}^{(\mathcal{N})}+\frac{2}{27}\left(1-\sum_{q=u, d, s} f_{T q}^{(\mathcal{N})}\right) \sum_{q=c, b, t} \frac{G_{q}}{m_{q}} .
$$

Using eq. (C.2), the above expression expands to

$$
\begin{aligned}
\frac{G_{\mathcal{N}}}{m_{\mathcal{N}}} & =\frac{g_{S} \sin \alpha \cos \alpha}{v_{0}}\left(\frac{1}{m_{h}^{2}}-\frac{1}{m_{H}^{2}}\right)\left[\sum_{q=u, d, s} f_{T q}^{(\mathcal{N})}+\frac{2}{9}\left(1-\sum_{q=u, d, s} f_{T q}^{(\mathcal{N})}\right)\right] \\
& =\frac{g_{S} \sin \alpha \cos \alpha}{v_{0}}\left(\frac{1}{m_{h}^{2}}-\frac{1}{m_{H}^{2}}\right) f_{\mathcal{N}},
\end{aligned}
$$

where

$$
f_{\mathcal{N}}=\frac{2}{9}+\frac{7}{9} \sum_{q=u, d, s} f_{T q}^{(\mathcal{N})}
$$

is the Higgs-nucleon coupling [96]. Thus, the effective DM-nucleon interaction Lagrangian can be written as

$$
\mathscr{L}_{\mathrm{DM}-\mathcal{N}}^{\mathrm{eff}}=-\sum_{\mathcal{N}=p, n} G_{\mathcal{N}} \bar{\psi} \psi \overline{\mathcal{N}} \mathcal{N}
$$

where

$$
G_{\mathcal{N}}=\frac{g_{S} \sin \alpha \cos \alpha}{v_{0}}\left(\frac{1}{m_{h}^{2}}-\frac{1}{m_{H}^{2}}\right) m_{\mathcal{N}} f_{\mathcal{N}}
$$

is the effective DM-nucleon coupling [87].

For a SI DM-nucleon interaction, the DM-nucleus interaction is a coherent sum over the total number of protons $Z$ and neutrons $(A-Z)$ in the target nucleus $N$. Thus, the SI DM-nucleus cross-section is given by

$$
\sigma_{\mathrm{SI}}^{\psi N}=\frac{\mu_{\psi N}^{2}}{\pi}\left[Z G_{p}+(A-Z) G_{n}\right]^{2}
$$

where $\mu_{\psi N}=m_{\psi} m_{N} /\left(m_{\psi}+m_{N}\right)$ is the DM-nucleus reduced mass.

\section{Effective potential}

We include the following 1-loop corrections to the zero temperature potential in the cutoff regularisation and on-shell scheme [20, 152]

$$
V_{1-\text { loop }}(\phi, S)=\sum_{i=\phi, S, \chi}^{W, Z, t, \psi} \frac{n_{i}}{64 \pi^{2}}\left[m_{i}^{4}\left(\log \frac{m_{i}^{2}}{m_{0 i}^{2}}-\frac{3}{2}\right)+2 m_{i}^{2} m_{0 i}^{2}\right],
$$

where $n_{\{\phi, S, \chi, W, Z, t, \psi\}}=\{1,1,3,6,3,-12,-4\}$. The subscript " 0 " implies that the particle masses are calculated at the $T=0$ minimum, i.e., $(\phi, S)=\left(v_{0}, s_{0}\right)$. The $\phi$ and $S$ field dependent masses are given in appendix $\mathrm{B}$, whereas the rest are given by

$$
\begin{array}{rlrl}
m_{W}^{2} & =\frac{g^{2}}{4} \phi^{2}, & m_{Z}^{2}=\frac{g^{2}+g^{\prime 2}}{4} \phi^{2}, & m_{t}^{2}=\frac{y_{t}^{2}}{2} \phi^{2}, \\
m_{\chi}^{2}=-\mu_{\Phi}^{2}+\lambda_{\Phi} \phi^{2}+\mu_{\Phi S} S+\frac{1}{2} \lambda_{\Phi S} S^{2}, & m_{\psi}=\mu_{\psi}+g_{S} S .
\end{array}
$$


The finite temperature corrections to the effective potential are given by

$$
V_{T}(\phi, S, T)=\frac{T^{4}}{2 \pi^{2}}\left[\sum_{i=\phi, S, \chi}^{W, Z} n_{i} J_{b}\left(\frac{m_{i}^{2}}{T^{2}}\right)+\sum_{i=t, \psi} n_{i} J_{f}\left(\frac{m_{i}^{2}}{T^{2}}\right)\right],
$$

where

$$
J_{b / f}\left(\frac{m_{i}^{2}}{T^{2}}\right)=\int_{0}^{\infty} d k k^{2} \log \left[1 \mp \exp \left(-\sqrt{\frac{k^{2}+m_{i}^{2}}{T^{2}}}\right)\right] .
$$

The final important correction comes from resumming the multi-loop contributions to the boson longitudinal polarizations which are infrared divergent [18, 153]. These are incorporate by supplementing the scalars and longitudinal polarizations of the gauge bosons with thermal mass corrections, in particular, by expanding eq. (D.3) to the leading order in $m^{2} / T^{2}$ [18]. For our model, they are given by

$$
\begin{aligned}
& \Pi_{\phi}(T)=\Pi_{\chi}(T)=T^{2}\left(\frac{g^{\prime 2}}{16}+\frac{3 g}{16}+\frac{\lambda_{\Phi}}{2}+\frac{y_{t}^{2}}{4}+\frac{\lambda_{\Phi S}}{24}\right), \\
& \Pi_{S}(T)=T^{2}\left(\frac{\lambda_{\Phi S}}{3}+\frac{\lambda_{S}}{4}+\frac{g_{S}^{2}}{6}\right), \quad \Pi_{W}(T)=\frac{11}{6} g^{2} T^{2} .
\end{aligned}
$$

For the $\phi$ and $S$ fields, the corrected masses are the eigenvalues of the following squared mass matrix

$$
\mathcal{M}^{2}+\left(\begin{array}{cc}
\Pi_{\phi}(T) & 0 \\
0 & \Pi_{S}(T)
\end{array}\right)
$$

where $\mathcal{M}^{2}$ is defined in eq. (A.10). For the $Z$ and $\gamma$ fields, namely $m_{Z / \gamma}^{2}+\Pi_{Z / \gamma}(T)$, the mass corrections are the eigenvalues of the following squared mass matrix

$$
\left(\begin{array}{cc}
\frac{1}{4} g^{2} \phi^{2}+\frac{11}{6} g^{2} T^{2} & -\frac{1}{4} g^{\prime} g \phi^{2} \\
-\frac{1}{4} g^{\prime} g \phi^{2} & \frac{1}{4} g^{\prime 2} \phi^{2}+\frac{11}{6} g^{\prime 2} T^{2}
\end{array}\right) .
$$

In other cases, we simply use the following substitution

$$
m_{i}^{2} \rightarrow m_{i}^{2}+\Pi_{i}
$$

Finally, the effective potential $V_{\text {eff }}(\phi, S, T)$ is given by

$$
V_{\text {eff }}(\phi, S, T)=V_{\text {tree }}(\phi, S)+V_{1-\text { loop }}(\phi, S)+V_{T}(\phi, S, T)
$$

where $V_{\text {tree }}(\phi, S)$ is the tree-level scalar potential in appendix A.

Open Access. This article is distributed under the terms of the Creative Commons Attribution License (CC-BY 4.0), which permits any use, distribution and reproduction in any medium, provided the original author(s) and source are credited. 


\section{References}

[1] ATLAS collaboration, Observation of a new particle in the search for the Standard Model Higgs boson with the ATLAS detector at the LHC, Phys. Lett. B 716 (2012) 1 [arXiv:1207.7214] [INSPIRE].

[2] CMS collaboration, Observation of a new boson at a mass of 125 GeV with the CMS experiment at the LHC, Phys. Lett. B 716 (2012) 30 [arXiv:1207.7235] [INSPIRE].

[3] LIGO ScIEnTIFIC and VIRGo collaborations, Observation of Gravitational Waves from a Binary Black Hole Merger, Phys. Rev. Lett. 116 (2016) 061102 [arXiv:1602.03837] [INSPIRE].

[4] LIGO SCIENTIFIC and VIRGo collaborations, GW151226: Observation of Gravitational Waves from a 22-Solar-Mass Binary Black Hole Coalescence, Phys. Rev. Lett. 116 (2016) 241103 [arXiv: 1606.04855] [INSPIRE].

[5] LIGO ScIENTIFIC and Virgo collaborations, First search for gravitational waves from known pulsars with Advanced LIGO, Astrophys. J. 839 (2017) 12 [Erratum ibid. 851 (2017) 71] [arXiv: 1701.07709] [INSPIRE].

[6] LIGO ScIEntific and Virgo collaborations, GW170104: Observation of a 50-Solar-Mass Binary Black Hole Coalescence at Redshift 0.2, Phys. Rev. Lett. 118 (2017) 221101 [Erratum ibid. 121 (2018) 129901] [arXiv:1706.01812] [INSPIRE].

[7] LIGO Scientific and VIrgo collaborations, GW170814: A Three-Detector Observation of Gravitational Waves from a Binary Black Hole Coalescence, Phys. Rev. Lett. 119 (2017) 141101 [arXiv:1709.09660] [INSPIRE].

[8] LIGO ScIENTIFIC and VIRGO collaborations, GW17081\%: Observation of Gravitational Waves from a Binary Neutron Star Inspiral, Phys. Rev. Lett. 119 (2017) 161101 [arXiv: 1710.05832] [INSPIRE].

[9] LiGO Scientific, Virgo, Fermi-GBM and INTEGRAL collaborations, Gravitational Waves and Gamma-rays from a Binary Neutron Star Merger: GW170817 and GRB 170817A, Astrophys. J. 848 (2017) L13 [arXiv:1710.05834] [INSPIRE].

[10] LIGO ScIEntific and ViRgo collaborations, GW170608: Observation of a 19-solar-mass Binary Black Hole Coalescence, Astrophys. J. 851 (2017) L35 [arXiv:1711.05578] [INSPIRE].

[11] C. Caprini et al., Science with the space-based interferometer eLISA. II: Gravitational waves from cosmological phase transitions, JCAP 04 (2016) 001 [arXiv: 1512.06239] [INSPIRE].

[12] D.J. Weir, Gravitational waves from a first order electroweak phase transition: a brief review, Phil. Trans. Roy. Soc. Lond. A 376 (2018) 20170126 [arXiv:1705.01783] [INSPIRE].

[13] C. Caprini and D.G. Figueroa, Cosmological Backgrounds of Gravitational Waves, Class. Quant. Grav. 35 (2018) 163001 [arXiv:1801.04268] [INSPIRE].

[14] V.A. Kuzmin, V.A. Rubakov and M.E. Shaposhnikov, On the Anomalous Electroweak Baryon Number Nonconservation in the Early Universe, Phys. Lett. B 155 (1985) 36 [INSPIRE].

[15] A.G. Cohen, D.B. Kaplan and A.E. Nelson, Progress in electroweak baryogenesis, Ann. Rev. Nucl. Part. Sci. 43 (1993) 27 [hep-ph/9302210] [INSPIRE]. 
[16] A. Riotto and M. Trodden, Recent progress in baryogenesis, Ann. Rev. Nucl. Part. Sci. 49 (1999) 35 [hep-ph/9901362] [INSPIRE].

[17] D.E. Morrissey and M.J. Ramsey-Musolf, Electroweak baryogenesis, New J. Phys. 14 (2012) 125003 [arXiv: 1206.2942] [INSPIRE].

[18] P.B. Arnold and O. Espinosa, The Effective potential and first order phase transitions: Beyond leading-order, Phys. Rev. D 47 (1993) 3546 [Erratum ibid. D 50 (1994) 6662] [hep-ph/9212235] [INSPIRE].

[19] K. Kajantie, M. Laine, K. Rummukainen and M.E. Shaposhnikov, A Nonperturbative analysis of the finite $T$ phase transition in $\mathrm{SU}(2) \times \mathrm{U}(1)$ electroweak theory, Nucl. Phys. B 493 (1997) 413 [hep-lat/9612006] [INSPIRE].

[20] D. Curtin, P. Meade and C.-T. Yu, Testing Electroweak Baryogenesis with Future Colliders, JHEP 11 (2014) 127 [arXiv:1409.0005] [INSPIRE].

[21] A.V. Kotwal, M.J. Ramsey-Musolf, J.M. No and P. Winslow, Singlet-catalyzed electroweak phase transitions in the $100 \mathrm{TeV}$ frontier, Phys. Rev. D 94 (2016) 035022 [arXiv: 1605.06123] [INSPIRE].

[22] J. Choi and R.R. Volkas, Real Higgs singlet and the electroweak phase transition in the Standard Model, Phys. Lett. B 317 (1993) 385 [hep-ph/9308234] [INSPIRE].

[23] A. Ashoorioon and T. Konstandin, Strong electroweak phase transitions without collider traces, JHEP 07 (2009) 086 [arXiv:0904.0353] [INSPIRE].

[24] K. Enqvist, S. Nurmi, T. Tenkanen and K. Tuominen, Standard Model with a real singlet scalar and inflation, JCAP 08 (2014) 035 [arXiv: 1407.0659] [INSPIRE].

[25] M. Kakizaki, S. Kanemura and T. Matsui, Gravitational waves as a probe of extended scalar sectors with the first order electroweak phase transition, Phys. Rev. D 92 (2015) 115007 [arXiv: 1509.08394] [INSPIRE].

[26] F.P. Huang, Y. Wan, D.-G. Wang, Y.-F. Cai and X. Zhang, Hearing the echoes of electroweak baryogenesis with gravitational wave detectors, Phys. Rev. D 94 (2016) 041702 [arXiv: 1601.01640] [INSPIRE].

[27] K. Hashino, M. Kakizaki, S. Kanemura and T. Matsui, Synergy between measurements of gravitational waves and the triple-Higgs coupling in probing the first-order electroweak phase transition, Phys. Rev. D 94 (2016) 015005 [arXiv: 1604.02069] [INSPIRE].

[28] M. Chala, G. Nardini and I. Sobolev, Unified explanation for dark matter and electroweak baryogenesis with direct detection and gravitational wave signatures, Phys. Rev. D 94 (2016) 055006 [arXiv: 1605.08663] [inSPIRE].

[29] T. Tenkanen, K. Tuominen and V. Vaskonen, A Strong Electroweak Phase Transition from the Inflaton Field, JCAP 09 (2016) 037 [arXiv: 1606.06063] [INSPIRE].

[30] A. Kobakhidze, A. Manning and J. Yue, Gravitational waves from the phase transition of a nonlinearly realized electroweak gauge symmetry, Int. J. Mod. Phys. D 26 (2017) 1750114 [arXiv: 1607.00883] [INSPIRE].

[31] P. Huang, A.J. Long and L.-T. Wang, Probing the Electroweak Phase Transition with Higgs Factories and Gravitational Waves, Phys. Rev. D 94 (2016) 075008 [arXiv:1608.06619] [INSPIRE]. 
[32] M. Artymowski, M. Lewicki and J.D. Wells, Gravitational wave and collider implications of electroweak baryogenesis aided by non-standard cosmology, JHEP 03 (2017) 066 [arXiv: 1609.07143] [INSPIRE].

[33] K. Hashino, M. Kakizaki, S. Kanemura, P. Ko and T. Matsui, Gravitational waves and Higgs boson couplings for exploring first order phase transition in the model with a singlet scalar field, Phys. Lett. B 766 (2017) 49 [arXiv: 1609.00297] [INSPIRE].

[34] V. Vaskonen, Electroweak baryogenesis and gravitational waves from a real scalar singlet, Phys. Rev. D 95 (2017) 123515 [arXiv:1611.02073] [INSPIRE].

[35] I. Baldes, Gravitational waves from the asymmetric-dark-matter generating phase transition, JCAP 05 (2017) 028 [arXiv:1702.02117] [INSPIRE].

[36] A. Beniwal, M. Lewicki, J.D. Wells, M. White and A.G. Williams, Gravitational wave, collider and dark matter signals from a scalar singlet electroweak baryogenesis, JHEP 08 (2017) 108 [arXiv: 1702.06124] [INSPIRE].

[37] A. Kobakhidze, C. Lagger, A. Manning and J. Yue, Gravitational waves from a supercooled electroweak phase transition and their detection with pulsar timing arrays, Eur. Phys. J. C 77 (2017) 570 [arXiv:1703.06552] [INSPIRE].

[38] R.-G. Cai, M. Sasaki and S.-J. Wang, The gravitational waves from the first-order phase transition with a dimension-six operator, JCAP 08 (2017) 004 [arXiv: 1707.03001] [INSPIRE].

[39] D. Croon, V. Sanz and G. White, Model Discrimination in Gravitational Wave spectra from Dark Phase Transitions, JHEP 08 (2018) 203 [arXiv: 1806.02332] [INSPIRE].

[40] I. Baldes and C. Garcia-Cely, Strong gravitational radiation from a simple dark matter model, arXiv: 1809.01198 [INSPIRE].

[41] K. Hashino, R. Jinno, M. Kakizaki, S. Kanemura, T. Takahashi and M. Takimoto, Fingerprinting models of first-order phase transitions by the synergy between collider and gravitational-wave experiments, arXiv:1809.04994 [INSPIRE].

[42] A. Ahriche, K. Hashino, S. Kanemura and S. Nasri, Gravitational Waves from Phase Transitions in Models with Charged Singlets, Phys. Lett. B 789 (2019) 119 [arXiv: 1809.09883] [INSPIRE].

[43] V. Silveira and A. Zee, Scalar phantoms, Phys. Lett. B 161 (1985) 136 [InSPIRE].

[44] J. McDonald, Gauge singlet scalars as cold dark matter, Phys. Rev. D 50 (1994) 3637 [hep-ph/0702143] [INSPIRE].

[45] C.P. Burgess, M. Pospelov and T. ter Veldhuis, The Minimal model of nonbaryonic dark matter: A Singlet scalar, Nucl. Phys. B 619 (2001) 709 [hep-ph/0011335] [InSPIRE].

[46] J.R. Espinosa, T. Konstandin, J.M. No and M. Quirós, Some Cosmological Implications of Hidden Sectors, Phys. Rev. D 78 (2008) 123528 [arXiv:0809.3215] [INSPIRE].

[47] T. Alanne, K. Tuominen and V. Vaskonen, Strong phase transition, dark matter and vacuum stability from simple hidden sectors, Nucl. Phys. B 889 (2014) 692 [arXiv: 1407.0688] [INSPIRE].

[48] V. Martín Lozano, J.M. Moreno and C.B. Park, Resonant Higgs boson pair production in the $h h \rightarrow b \bar{b} W W \rightarrow b \bar{b} \ell^{+} \nu \ell^{-} \bar{\nu}$ decay channel, JHEP 08 (2015) 004 [arXiv:1501.03799] [INSPIRE]. 
[49] A. Falkowski, C. Gross and O. Lebedev, A second Higgs from the Higgs portal, JHEP 05 (2015) 057 [arXiv: 1502.01361] [inSPIRE].

[50] D. Buttazzo, F. Sala and A. Tesi, Singlet-like Higgs bosons at present and future colliders, JHEP 11 (2015) 158 [arXiv:1505.05488] [INSPIRE].

[51] M. Heikinheimo, T. Tenkanen, K. Tuominen and V. Vaskonen, Observational Constraints on Decoupled Hidden Sectors, Phys. Rev. D 94 (2016) 063506 [Erratum ibid. D 96 (2017) 109902] [arXiv: 1604.02401] [INSPIRE].

[52] C. Balázs, A. Fowlie, A. Mazumdar and G. White, Gravitational waves at aLIGO and vacuum stability with a scalar singlet extension of the Standard Model, Phys. Rev. D 95 (2017) 043505 [arXiv: 1611.01617] [INSPIRE].

[53] I.M. Lewis and M. Sullivan, Benchmarks for Double Higgs Production in the Singlet Extended Standard Model at the LHC, Phys. Rev. D 96 (2017) 035037 [arXiv:1701.08774] [INSPIRE].

[54] P.H. Ghorbani, Electroweak Baryogenesis and Dark Matter via a Pseudoscalar vs. Scalar, JHEP 08 (2017) 058 [arXiv:1703.06506] [INSPIRE].

[55] C.-Y. Chen, J. Kozaczuk and I.M. Lewis, Non-resonant Collider Signatures of a Singlet-Driven Electroweak Phase Transition, JHEP 08 (2017) 096 [arXiv:1704.05844] [INSPIRE].

[56] T. Kamon, P. Ko and J. Li, Characterizing Higgs portal dark matter models at the ILC, Eur. Phys. J. C 77 (2017) 652 [arXiv:1705.02149] [InSPIRE].

[57] M. Ettefaghi and R. Moazzemi, Analyzing of singlet fermionic dark matter via the updated direct detection data, Eur. Phys. J. C 77 (2017) 343 [arXiv: 1705.07571] [inSPIRE].

[58] M.J. Baker, M. Breitbach, J. Kopp and L. Mittnacht, Dynamic Freeze-In: Impact of Thermal Masses and Cosmological Phase Transitions on Dark Matter Production, JHEP 03 (2018) 114 [arXiv: 1712.03962] [INSPIRE].

[59] S. Baum, M. Carena, N.R. Shah and C.E.M. Wagner, Higgs portals for thermal Dark Matter. EFT perspectives and the NMSSM, JHEP 04 (2018) 069 [arXiv:1712.09873] [INSPIRE].

[60] N. Bernal, C. Cosme and T. Tenkanen, Phenomenology of Self-Interacting Dark Matter in a Matter-Dominated Universe, Eur. Phys. J. C 79 (2019) 99 [arXiv:1803.08064] [INSPIRE].

[61] Y.G. Kim, K.Y. Lee and S. Shin, Singlet fermionic dark matter, JHEP 05 (2008) 100 [arXiv: 0803.2932] [INSPIRE].

[62] S. Baek, P. Ko and W.-I. Park, Search for the Higgs portal to a singlet fermionic dark matter at the LHC, JHEP 02 (2012) 047 [arXiv:1112.1847] [INSPIRE].

[63] S. Baek, P. Ko, W.-I. Park and E. Senaha, Vacuum structure and stability of a singlet fermion dark matter model with a singlet scalar messenger, JHEP 11 (2012) 116 [arXiv: 1209.4163] [INSPIRE].

[64] J.R. Espinosa, T. Konstandin and F. Riva, Strong Electroweak Phase Transitions in the Standard Model with a Singlet, Nucl. Phys. B 854 (2012) 592 [arXiv:1107.5441] [inSPIRE].

[65] M. Fairbairn and R. Hogan, Singlet Fermionic Dark Matter and the Electroweak Phase Transition, JHEP 09 (2013) 022 [arXiv: 1305.3452] [INSPIRE]. 
[66] T. Li and Y.-F. Zhou, Strongly first order phase transition in the singlet fermionic dark matter model after LUX, JHEP 07 (2014) 006 [arXiv:1402.3087] [INSPIRE].

[67] Planck collaboration, Planck 2015 results. XIII. Cosmological parameters, Astron. Astrophys. 594 (2016) A13 [arXiv:1502.01589] [INSPIRE].

[68] XENON collaboration, Dark Matter Search Results from a One Ton-Year Exposure of XENON1T, Phys. Rev. Lett. 121 (2018) 111302 [arXiv:1805.12562] [INSPIRE].

[69] J. Haller, A. Hoecker, R. Kogler, K. Mönig, T. Peiffer and J. Stelzer, Update of the global electroweak fit and constraints on two-Higgs-doublet models, Eur. Phys. J. C 78 (2018) 675 [arXiv: 1803.01853] [INSPIRE].

[70] P. Bechtle et al., HiggsBounds-4: Improved Tests of Extended Higgs Sectors against Exclusion Bounds from LEP, the Tevatron and the LHC, Eur. Phys. J. C 74 (2014) 2693 [arXiv: 1311.0055] [INSPIRE].

[71] P. Bechtle, S. Heinemeyer, O. Stål, T. Stefaniak and G. Weiglein, HiggsSignals: Confronting arbitrary Higgs sectors with measurements at the Tevatron and the LHC, Eur. Phys. J. C 74 (2014) 2711 [arXiv:1305.1933] [InSPIRE].

[72] J.M. Cline, K. Kainulainen, P. Scott and C. Weniger, Update on scalar singlet dark matter, Phys. Rev. D 88 (2013) 055025 [Erratum ibid. D 92 (2015) 039906] [arXiv:1306.4710] [INSPIRE].

[73] A. Beniwal et al., Combined analysis of effective Higgs portal dark matter models, Phys. Rev. D 93 (2016) 115016 [arXiv: 1512.06458] [INSPIRE].

[74] X.-G. He and J. Tandean, New LUX and PandaX-II Results Illuminating the Simplest Higgs-Portal Dark Matter Models, JHEP 12 (2016) 074 [arXiv: 1609.03551] [INSPIRE].

[75] M. Escudero, A. Berlin, D. Hooper and M.-X. Lin, Toward (Finally!) Ruling Out Z and Higgs Mediated Dark Matter Models, JCAP 12 (2016) 029 [arXiv:1609.09079] [INSPIRE].

[76] H. Wu and S. Zheng, Scalar Dark Matter: Real vs Complex, JHEP 03 (2017) 142 [arXiv: 1610.06292] [INSPIRE].

[77] S. Banerjee and N. Chakrabarty, A revisit to scalar dark matter with radiative corrections, arXiv: 1612.01973 [INSPIRE].

[78] J.A. Casas, D.G. Cerdeño, J.M. Moreno and J. Quilis, Reopening the Higgs portal for single scalar dark matter, JHEP 05 (2017) 036 [arXiv: 1701.08134] [INSPIRE].

[79] GAMBIT collaboration, Status of the scalar singlet dark matter model, Eur. Phys. J. C 77 (2017) 568 [arXiv: 1705.07931] [INSPIRE].

[80] M. Hoferichter, P. Klos, J. Menéndez and A. Schwenk, Improved limits for Higgs-portal dark matter from LHC searches, Phys. Rev. Lett. 119 (2017) 181803 [arXiv:1708.02245] [INSPIRE].

[81] P. Athron, J.M. Cornell, F. Kahlhoefer, J. McKay, P. Scott and S. Wild, Impact of vacuum stability, perturbativity and XENON1T on global fits of $\mathbb{Z}_{2}$ and $\mathbb{Z}_{3}$ scalar singlet dark matter, Eur. Phys. J. C 78 (2018) 830 [arXiv:1806.11281] [INSPIRE].

[82] A. Semenov, LanHEP - A package for automatic generation of Feynman rules from the Lagrangian. Version 3.2, Comput. Phys. Commun. 201 (2016) 167 [arXiv:1412.5016] [INSPIRE]. 
[83] G. Bélanger, F. Boudjema, A. Pukhov and A. Semenov, MicrOMEGAs4.1: two dark matter candidates, Comput. Phys. Commun. 192 (2015) 322 [arXiv:1407.6129] [INSPIRE].

[84] A. Belyaev, N.D. Christensen and A. Pukhov, CalcHEP 3.4 for collider physics within and beyond the Standard Model, Comput. Phys. Commun. 184 (2013) 1729 [arXiv:1207.6082] [INSPIRE].

[85] GAMBIT collaboration, Comparison of statistical sampling methods with ScannerBit, the GAMBIT scanning module, Eur. Phys. J. C 77 (2017) 761 [arXiv:1705. 07959] [InSPIRE].

[86] J.M. Cline and K. Kainulainen, Electroweak baryogenesis and dark matter from a singlet Higgs, JCAP 01 (2013) 012 [arXiv:1210.4196] [INSPIRE].

[87] P. Agrawal, Z. Chacko, C. Kilic and R.K. Mishra, A Classification of Dark Matter Candidates with Primarily Spin-Dependent Interactions with Matter, arXiv:1003.1912 [INSPIRE].

[88] S. Esch, M. Klasen and C.E. Yaguna, Detection prospects of singlet fermionic dark matter, Phys. Rev. D 88 (2013) 075017 [arXiv: 1308.0951] [InSPIRE].

[89] Z. Bagherian, M.M. Ettefaghi, Z. Haghgouyan and R. Moazzemi, A new parameter space study of the fermionic cold dark matter model, JCAP 10 (2014) 033 [arXiv:1406.2927] [INSPIRE].

[90] T.H. Franarin, C.A.Z. Vasconcellos and D. Hadjimichef, On the possibility of a $130 \mathrm{GeV}$ gamma-ray line from annihilating singlet fermionic dark matter, Astron. Nachr. 335 (2014) 647 [arXiv : 1404.0406] [INSPIRE].

[91] Y.G. Kim, K.Y. Lee, C.B. Park and S. Shin, Secluded singlet fermionic dark matter driven by the Fermi gamma-ray excess, Phys. Rev. D 93 (2016) 075023 [arXiv:1601.05089] [INSPIRE].

[92] K. Ghorbani, Fermionic dark matter with pseudo-scalar Yukawa interaction, JCAP 01 (2015) 015 [arXiv: 1408.4929] [INSPIRE].

[93] C. Balázs, T. Li, C. Savage and M. White, Interpreting the Fermi-LAT gamma ray excess in the simplified framework, Phys. Rev. D 92 (2015) 123520 [arXiv:1505. 06758] [InSPIRE].

[94] GAMBIT collaboration, Global analyses of Higgs portal singlet dark matter models using GAMBIT, Eur. Phys. J. C 79 (2019) 38 [arXiv:1808.10465] [INSPIRE].

[95] Y.G. Kim, C.B. Park and S. Shin, Collider probes of singlet fermionic dark matter scenarios for the Fermi gamma-ray excess, JHEP 12 (2018) 036 [arXiv:1809.01143] [INSPIRE].

[96] The Gambit Dark Matter Workgroup collaboration, DarkBit: A GAMBIT module for computing dark matter observables and likelihoods, Eur. Phys. J. C 77 (2017) 831 [arXiv:1705.07920] [INSPIRE].

[97] C. Grojean and G. Servant, Gravitational Waves from Phase Transitions at the Electroweak Scale and Beyond, Phys. Rev. D 75 (2007) 043507 [hep-ph/0607107] [INSPIRE].

[98] M. Quirós, Finite temperature field theory and phase transitions, in Proceedings of Summer School in High-energy physics and cosmology, Trieste Italy (1998), pg. 187 [hep-ph/9901312] [INSPIRE].

[99] K. Funakubo and E. Senaha, Electroweak phase transition, critical bubbles and sphaleron decoupling condition in the MSSM, Phys. Rev. D 79 (2009) 115024 [arXiv:0905.2022] [INSPIRE]. 
[100] A. Katz and M. Perelstein, Higgs Couplings and Electroweak Phase Transition, JHEP 07 (2014) 108 [arXiv: 1401.1827] [INSPIRE].

[101] K. Fuyuto and E. Senaha, Improved sphaleron decoupling condition and the Higgs coupling constants in the real singlet-extended standard model, Phys. Rev. D 90 (2014) 015015 [arXiv: 1406.0433] [INSPIRE].

[102] J. Ellis, M. Lewicki and J.M. No, On the Maximal Strength of a First-Order Electroweak Phase Transition and its Gravitational Wave Signal, Submitted to: JCAP (2018) [arXiv: 1809.08242] [INSPIRE].

[103] D. Bödeker and G.D. Moore, Can electroweak bubble walls run away?, JCAP 05 (2009) 009 [arXiv: 0903.4099] [INSPIRE].

[104] J. Kozaczuk, Bubble Expansion and the Viability of Singlet-Driven Electroweak Baryogenesis, JHEP 10 (2015) 135 [arXiv: 1506. 04741] [INSPIRE].

[105] G. Kurup and M. Perelstein, Dynamics of Electroweak Phase Transition In Singlet-Scalar Extension of the Standard Model, Phys. Rev. D 96 (2017) 015036 [arXiv:1704.03381] [INSPIRE].

[106] J.M. No, Large Gravitational Wave Background Signals in Electroweak Baryogenesis Scenarios, Phys. Rev. D 84 (2011) 124025 [arXiv:1103.2159] [InSPIRE].

[107] C. Caprini and J.M. No, Supersonic Electroweak Baryogenesis: Achieving Baryogenesis for Fast Bubble Walls, JCAP 01 (2012) 031 [arXiv:1111.1726] [InSPIRE].

[108] A. Katz and A. Riotto, Baryogenesis and Gravitational Waves from Runaway Bubble Collisions, JCAP 11 (2016) 011 [arXiv:1608.00583] [INSPIRE].

[109] M.E. Peskin and T. Takeuchi, Estimation of oblique electroweak corrections, Phys. Rev. D 46 (1992) 381 [INSPIRE].

[110] W. Grimus, L. Lavoura, O.M. Ogreid and P. Osland, The Oblique parameters in multi-Higgs-doublet models, Nucl. Phys. B 801 (2008) 81 [arXiv:0802.4353] [INSPIRE].

[111] S. Profumo, M.J. Ramsey-Musolf, C.L. Wainwright and P. Winslow, Singlet-catalyzed electroweak phase transitions and precision Higgs boson studies, Phys. Rev. D 91 (2015) 035018 [arXiv: 1407.5342] [INSPIRE].

[112] A. Djouadi, The Anatomy of electro-weak symmetry breaking. I: The Higgs boson in the standard model, Phys. Rept. 457 (2008) 1 [hep-ph/0503172] [INSPIRE].

[113] ATLAS collaboration, Measurements of the Higgs boson production and decay rates and coupling strengths using pp collision data at $\sqrt{s}=7$ and $8 \mathrm{TeV}$ in the ATLAS experiment, Eur. Phys. J. C 76 (2016) 6 [arXiv:1507.04548] [inSPIRE].

[114] CMS collaboration, Measurement of Higgs boson production and properties in the $W W$ decay channel with leptonic final states, JHEP 01 (2014) 096 [arXiv:1312.1129] [INSPIRE].

[115] CMS collaboration, Measurement of the properties of a Higgs boson in the four-lepton final state, Phys. Rev. D 89 (2014) 092007 [arXiv:1312.5353] [INSPIRE].

[116] CMS collaboration, Observation of the diphoton decay of the Higgs boson and measurement of its properties, Eur. Phys. J. C 74 (2014) 3076 [arXiv:1407.0558] [INSPIRE].

[117] CMS collaboration, Evidence for the direct decay of the $125 \mathrm{GeV}$ Higgs boson to fermions, Nature Phys. 10 (2014) 557 [arXiv:1401.6527] [INSPIRE]. 
[118] O. Stål and T. Stefaniak, Constraining extended Higgs sectors with HiggsSignals, PoS (EPS-HEP2013) 314 [arXiv: 1310.4039] [INSPIRE].

[119] G. Cowan, K. Cranmer, E. Gross and O. Vitells, Asymptotic formulae for likelihood-based tests of new physics, Eur. Phys. J. C 71 (2011) 1554 [Erratum ibid. C 73 (2013) 2501] [arXiv: 1007.1727] [INSPIRE].

[120] S.S. Wilks, The Large-Sample Distribution of the Likelihood Ratio for Testing Composite Hypotheses, Ann. Math. Statist. 9 (1938) 60.

[121] P. Scott, Pippi - painless parsing, post-processing and plotting of posterior and likelihood samples, Eur. Phys. J. Plus 127 (2012) 138 [arXiv:1206.2245] [INSPIRE].

[122] LUX-ZEPLIN collaboration, Projected WIMP Sensitivity of the LUX-ZEPLIN (LZ) Dark Matter Experiment, arXiv:1802.06039 [INSPIRE].

[123] M.S. Turner, E.J. Weinberg and L.M. Widrow, Bubble nucleation in first order inflation and other cosmological phase transitions, Phys. Rev. D 46 (1992) 2384 [InSPIRE].

[124] A.H. Guth and E.J. Weinberg, Could the Universe Have Recovered from a Slow First Order Phase Transition?, Nucl. Phys. B 212 (1983) 321 [InSPIRE].

[125] K. Enqvist, J. Ignatius, K. Kajantie and K. Rummukainen, Nucleation and bubble growth in a first order cosmological electroweak phase transition, Phys. Rev. D 45 (1992) 3415 [INSPIRE].

[126] M. Kamionkowski, A. Kosowsky and M.S. Turner, Gravitational radiation from first order phase transitions, Phys. Rev. D 49 (1994) 2837 [astro-ph/9310044] [INSPIRE].

[127] S.J. Huber and T. Konstandin, Gravitational Wave Production by Collisions: More Bubbles, JCAP 09 (2008) 022 [arXiv:0806.1828] [INSPIRE].

[128] R. Jinno and M. Takimoto, Gravitational waves from bubble collisions: An analytic derivation, Phys. Rev. D 95 (2017) 024009 [arXiv: 1605.01403] [INSPIRE].

[129] R. Jinno and M. Takimoto, Gravitational waves from bubble dynamics: Beyond the Envelope, JCAP 01 (2019) 060 [arXiv:1707.03111] [INSPIRE].

[130] D. Bödeker and G.D. Moore, Electroweak Bubble Wall Speed Limit, JCAP 05 (2017) 025 [arXiv: 1703.08215] [INSPIRE].

[131] M. Hindmarsh, S.J. Huber, K. Rummukainen and D.J. Weir, Gravitational waves from the sound of a first order phase transition, Phys. Rev. Lett. 112 (2014) 041301

[arXiv: 1304 .2433] [INSPIRE].

[132] M. Hindmarsh, S.J. Huber, K. Rummukainen and D.J. Weir, Numerical simulations of acoustically generated gravitational waves at a first order phase transition, Phys. Rev. D 92 (2015) 123009 [arXiv: 1504.03291] [inSPIRE].

[133] M. Hindmarsh, Sound shell model for acoustic gravitational wave production at a first-order phase transition in the early Universe, Phys. Rev. Lett. 120 (2018) 071301 [arXiv: 1608.04735] [INSPIRE].

[134] M. Hindmarsh, S.J. Huber, K. Rummukainen and D.J. Weir, Shape of the acoustic gravitational wave power spectrum from a first order phase transition, Phys. Rev. D 96 (2017) 103520 [arXiv:1704.05871] [INSPIRE]. 
[135] C. Caprini, R. Durrer and G. Servant, The stochastic gravitational wave background from turbulence and magnetic fields generated by a first-order phase transition, JCAP 12 (2009) 024 [arXiv:0909.0622] [inSPIRE].

[136] A. Kosowsky, A. Mack and T. Kahniashvili, Gravitational radiation from cosmological turbulence, Phys. Rev. D 66 (2002) 024030 [astro-ph/0111483] [INSPIRE].

[137] G. Gogoberidze, T. Kahniashvili and A. Kosowsky, The Spectrum of Gravitational Radiation from Primordial Turbulence, Phys. Rev. D 76 (2007) 083002 [arXiv: 0705.1733] [InSPIRE].

[138] P. Niksa, M. Schlederer and G. Sigl, Gravitational Waves produced by Compressible MHD Turbulence from Cosmological Phase Transitions, Class. Quant. Grav. 35 (2018) 144001 [arXiv: 1803.02271] [INSPIRE].

[139] N. Bartolo et al., Science with the space-based interferometer LISA. IV: Probing inflation with gravitational waves, JCAP 12 (2016) 026 [arXiv:1610.06481] [INSPIRE].

[140] K. Yagi and N. Seto, Detector configuration of DECIGO/BBO and identification of cosmological neutron-star binaries, Phys. Rev. D 83 (2011) 044011 [Erratum ibid. D 95 (2017) 109901] [arXiv: 1101.3940] [INSPIRE].

[141] LIGO Scientific collaboration, Advanced LIGO, Class. Quant. Grav. 32 (2015) 074001 [arXiv: 1411.4547] [INSPIRE].

[142] LIGO Scientific and VIRGO collaborations, GW150914: Implications for the stochastic gravitational wave background from binary black holes, Phys. Rev. Lett. 116 (2016) 131102 [arXiv: 1602.03847] [INSPIRE].

[143] E. Thrane and J.D. Romano, Sensitivity curves for searches for gravitational-wave backgrounds, Phys. Rev. D 88 (2013) 124032 [arXiv:1310.5300] [InSPIRE].

[144] R. van Haasteren et al., Placing limits on the stochastic gravitational-wave background using European Pulsar Timing Array data, Mon. Not. Roy. Astron. Soc. 414 (2011) 3117 [arXiv: 1103.0576].

[145] G. Janssen et al., Gravitational wave astronomy with the SKA, PoS (AASKA14) 037 [arXiv: 1501.00127] [INSPIRE].

[146] LIGO Scientific collaboration, Exploring the Sensitivity of Next Generation Gravitational Wave Detectors, Class. Quant. Grav. 34 (2017) 044001 [arXiv: 1607.08697] [INSPIRE].

[147] M. Punturo et al., The Einstein Telescope: A third-generation gravitational wave observatory, Class. Quant. Grav. 27 (2010) 194002 [INSPIRE].

[148] S. Hild et al., Sensitivity Studies for Third-Generation Gravitational Wave Observatories, Class. Quant. Grav. 28 (2011) 094013 [arXiv: 1012.0908] [INSPIRE].

[149] J. Ellis, TikZ-Feynman: Feynman diagrams with TikZ, Comput. Phys. Commun. 210 (2017) 103 [arXiv: 1601.05437] [InSPIRE].

[150] A. Berlin, D. Hooper and S.D. McDermott, Simplified Dark Matter Models for the Galactic Center Gamma-Ray Excess, Phys. Rev. D 89 (2014) 115022 [arXiv: 1404.0022] [InSPIRE].

[151] M.A. Shifman, A.I. Vainshtein and V.I. Zakharov, Remarks on Higgs Boson Interactions with Nucleons, Phys. Lett. B 78 (1978) 443 [INSPIRE].

[152] C. Delaunay, C. Grojean and J.D. Wells, Dynamics of Non-renormalizable Electroweak Symmetry Breaking, JHEP 04 (2008) 029 [arXiv:0711.2511] [InSPIRE].

[153] M.E. Carrington, The Effective potential at finite temperature in the Standard Model, Phys. Rev. D 45 (1992) 2933 [INSPIRE]. 